This item was submitted to Loughborough's Research Repository by the author.

Items in Figshare are protected by copyright, with all rights reserved, unless otherwise indicated.

\title{
Remote vibration measurements: compensation of waveform distortion due to whole body translations
}

PLEASE CITE THE PUBLISHED VERSION

http://dx.doi.org/10.1006/jsvi.1998.1529

\section{PUBLISHER}

Elsevier (@ Academic Press)

VERSION

AM (Accepted Manuscript)

\section{LICENCE}

CC BY-NC-ND 4.0

\section{REPOSITORY RECORD}

Hocknell, Alan, Roy Jones, and Steve Rothberg. 2019. "Remote Vibration Measurements: Compensation of Waveform Distortion Due to Whole Body Translations". figshare. https://hdl.handle.net/2134/9662. 
This item was submitted to Loughborough's Institutional Repository (https://dspace.lboro.ac.uk/) by the author and is made available under the following Creative Commons Licence conditions.

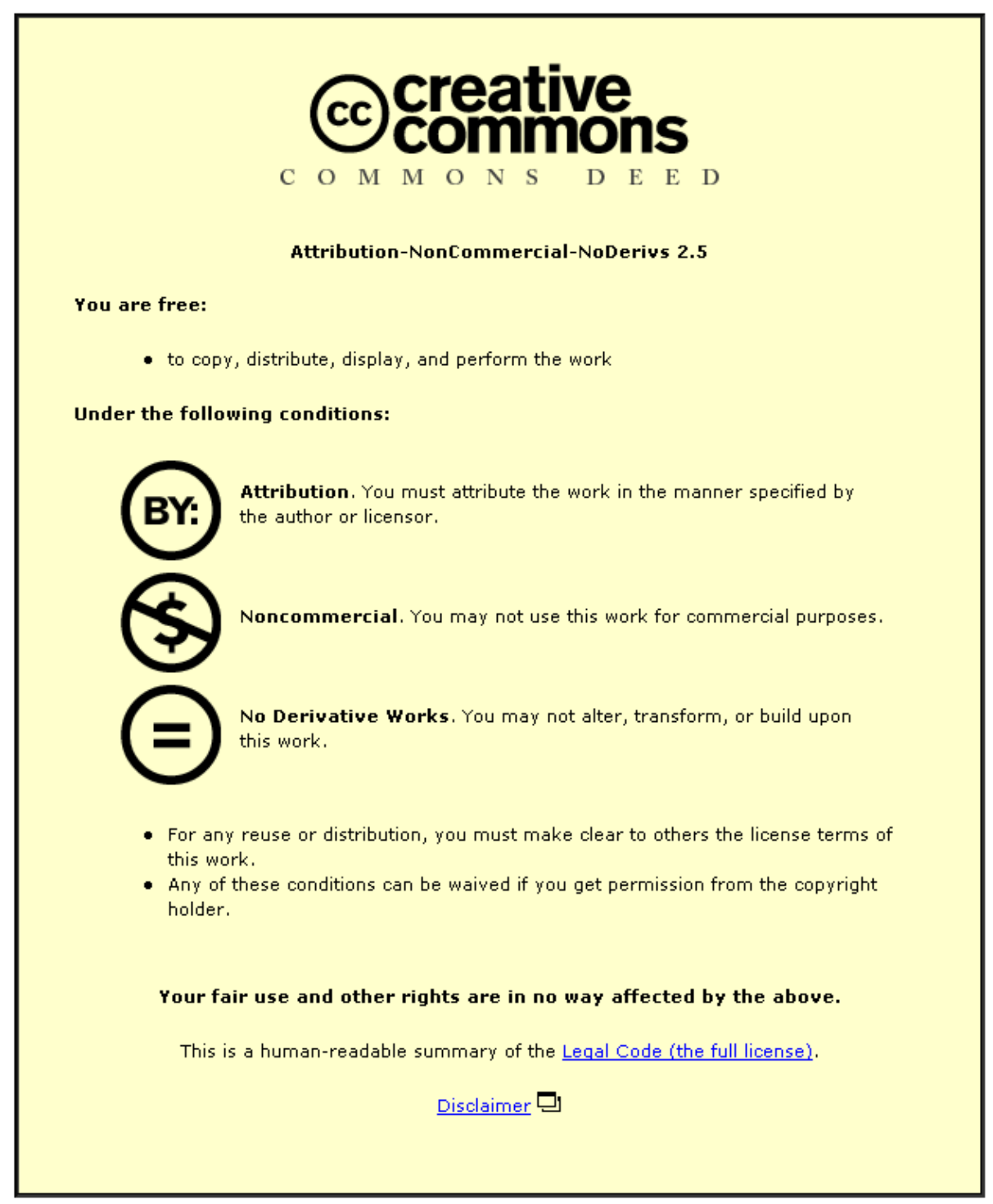

For the full text of this licence, please go to: http://creativecommons.org/licenses/by-nc-nd/2.5/ 

WAVEFORM DISTORTION DUE TO WHOLE BODY TRANSLATIONS

\author{
A.Hocknell, R.Jones \& S.J.Rothberg \\ Loughborough University \\ Leicestershire, LE11 3TU, UK
}

Short Title : Remote vibration measurements

Total pages : 30

Total figures : 18

Correspondence to :

Dr Steve Rothberg

Department of Mechanical Engineering

Loughborough University

Loughborough, Leicestershire

LE11 3TU, UK

Tel. 01509223245

e-mail : s.j.rothberg@lboro.ac.uk 


\begin{abstract}
Remote vibration measurements using the laser Doppler technique are a practical and increasingly popular alternative to the use of contacting transducers. This study concerns aspects of non-contact measurements that result when the vibrating target additionally undergoes larger scale whole body translation. The characteristics of a single remote measurement of steady state vibration on a body undergoing whole body translation are investigated in both the time and frequency domains, where waveform distortion and whole body target displacement induced harmonics are observed. A technique which compensates for the relative whole body displacement between the target and a remote transducer is introduced, in which two simultaneous remote measurements are combined to derive a closer estimate of the measurement which would be made by a contacting transducer attached to the target surface. A substantial improvement in the data quality obtainable from a single remote measurement is demonstrated using a numerical simulation and in experimental data captured remotely from a golf club head during and immediately following an impact with a golf ball. Remote measurements are particularly suited to analysis of impacting bodies and the displacement compensation technique is thus developed in this paper to investigate transient pulse propagation effects. The improvement in data quality obtained is demonstrated using data pertaining to the deformation of a golf ball during an impact in which the whole ball accelerates forwards rapidly.
\end{abstract}




\section{INTRODUCTION}

Vibration response measurements taken under actual operating conditions are important for many reasons including product development or refinement and the validation of computational analysis. Measurements of this nature can however be complicated by large scale, whole body motion of the subject under investigation. Traditionally, contacting transducers fixed to the body are used to obtain point vibration measurements. However, measurement situations can arise in which a contacting transducer may be damaged during the whole body motion, such as in an impact, or where the low mass of the body causes the mass of a contacting transducer to be significant. Additionally, complicated or large scale movement of the body introduces difficulties in signal retrieval by electrical connection.

Remote point vibration measurements using the laser Doppler technique are a practical and increasingly popular alternative to the use of contacting transducers in difficult measurement situations. All remote measurements, however, operate from a fundamentally different measurement perspective in which the transducer is fixed in space, rather than to a point on the target body. This study concerns aspects of such non-contact measurements that result when the target undergoes whole body translation. In these measurements the recorded data is taken not from a single point but from a continuous succession of points on the target body such that data at the end of a measurement may originate from a point whose separation from the original interrogated region is many times the size of the instantaneously probed region. In particular, this paper investigates remote point vibration measurements in the presence of larger scale whole body motion using a laser Doppler vibrometer. For this 
transducer, a body is deemed to have 'moved' if it is displaced by more than one laser beam diameter, typically $0.1-1 \mathrm{~mm}$, in a direction perpendicular to the sensing axis of the vibrometer since this causes data at each end of the measurement to originate from entirely distinct points on the target surface.

A method which compensates for relative whole body displacement between the target and a remote transducer fixed in space is introduced. This displacement compensation technique uses data from two simultaneous remote measurements combined with a measurement of the whole body target displacement to produce a much closer estimate in time of the measurement which would have been made by a transducer fixed to a point on the body. The combined measurement 'exists' for the time in which the target moves between the two remote laser beams and represents a significant improvement on the quality of data from a single remote measurement, as demonstrated in this study by application of the technique to simulated steady state vibration on a plate which translates with several velocity profiles. The improvement in data quality obtainable from practical remote laser Doppler vibrometer measurements is illustrated using data captured from a lightweight hollow golf club head, which translates a distance of approximately $10 \mathrm{~cm}$ and exhibits steady state vibration in the first few milliseconds after impact.

The investigation of lightweight bodies during impact is an example of another difficult measurement situation to which remote measurements are particularly suited. However, the transient vibration phenomena which occur in an impact are generally accompanied by rapid whole body acceleration. The displacement compensation technique is thus developed in this study to enable analysis of transient pulse 
propagation through a moving body using data from remote measurements. Data captured from a golf ball during impact with a golf club is used as an example, since the whole ball is displaced by approximately $11 \mathrm{~mm}$ as it accelerates from rest to $55 \mathrm{~ms}^{-1}$ during the $450 \mu \mathrm{s}$ impact whilst simultaneously exhibiting smaller scale elastic deformations of interest. Application of the displacement compensation technique illustrates how remote measurements can be processed to approximate closely data which would be recorded from a transducer fixed to the moving target body.

\section{REMOTE VIBRATION MEASUREMENTS ON MOVING BODIES}

The laser Doppler vibrometer is a non-contacting velocity transducer which is often employed in vibration measurement systems where the target body is hot, light or rotating. The vibrometer focuses a small beam of coherent light on the target to measure the component of target velocity which lies along the axis of the incident laser beam, as detailed in [1]. If normal-to-surface vibration velocity components are of primary interest then alignment of the laser beam perpendicular to the surface ensures in-plane velocity components due to either vibration or whole body motion will not contribute to the measured signal. This leaves a signal containing vibration information from a continuous succession of points on the target but which does not include components of whole body velocity associated with the changing probed region on the target. In this study, the only whole body motion considered is perpendicular to the direction of incidence of the probe laser beam and the whole body displacement in this direction must be measured as part of the displacement compensation technique. The required accuracy of this measurement is of the order of one laser beam diameter, 
therefore only low frequency components of the signal are needed to define the whole body motion satisfactorily. This being the case, the whole body displacement measurement can be taken from any convenient point on the deforming body and the signal then smoothed at an appropriate cut-off frequency. Experience has shown that the required data is easily obtained and this method works well as part of the displacement compensation process, improving the data quality in a range of different conditions.

Remote vibration measurements have been made previously on targets rotating about a fixed axis. A fixed point on a target rotating in a plane perpendicular to a laser vibrometer can be followed by continuous circular scanning of the laser beam in synchronisation with the target rotation speed. A technique for non-synchronous rotation of the target and laser beam has also been introduced for frequency domain analysis of rotating bodies [2]. Continuous scanning could be similarly applied to a simultaneously vibrating and translating target body, provided the required scanning motion was accurately known and did not exceed the maximum beam scan rate. However, it is impractical to synchronise the scan of a beam to the large and often non-linear accelerations on impacted bodies and non-synchronous scanning would add undue complexity to the measurement. The technique described in this paper in fact addresses the converse of the scanning beam case in which translation of the target perpendicular to the stationary laser beam causes data to be recorded from a spatially dense succession of points on the target surface. 


\section{A SINGLE REMOTE MEASUREMENT OF STEADY STATE}

\section{VIBRATION ON A TRANSLATING BODY}

\subsection{TIME DOMAIN CHARACTERISTICS}

Figure 1a shows a probe laser beam from remote point $A_{R}$ aligned perpendicular to the direction of whole body motion such that the initially probed region on the target, $A_{F}$, is a distance $x_{A}$ from an arbitrary reference point on the target, taken as the target edge in this example. The target shown, whose vibration is to be simulated, is a flat square plate of side $l$, simply supported on all sides and vibrating with angular frequency $\omega$. (The simply supported boundary condition is considered in order to simplify the analytical description of the experimental techniques presented in this paper, which are of general applicability). In the absence of whole body translation in the $x y$-plane the vibration response $V_{A F}(t)$ measured by either a fixed or a remote transducer at point $A_{F}$ is given by :

$$
V_{A F}(t)=A \sin (\omega t) \sin \left(n \pi x_{A} / l\right)
$$

where $A$ is the amplitude of vibration of the plate and $n$ is the order of the vibration mode. If the plate translates with velocity $\dot{x}(t)$, as shown in Figure $1 \mathrm{~b}$, the response, $V_{A R}(t)$, measured by the remote transducer becomes modulated and is given by :

$$
V_{A R}(t)=A \sin (\omega t) \sin \left(\frac{n \pi}{l}\left(x_{A}-\int_{0}^{t} \dot{x} d t\right)\right)
$$

In this case, $V_{A F}(t)$ continues to represent the measurement made by a transducer fixed to point $A_{F}$ on the target. The time dependence of the second sine term in equation (2), however, indicates how the remote measurement $V_{A R}(t)$ will comprise vibration response data from a spatially dense succession of points across the target. When compared to $V_{A F}(t)$, the $V_{A R}(t)$ waveform is thus both distorted from the original 
sinusoidal shape in time and has an amplitude which is modulated in the range 0-A according to the terms in the second sine expression of equation (2). This is simulated in Figure 2, where $V_{A F}(t)$ and $V_{A R}(t)$ are plotted for a time period corresponding to the passage of the entire plate past a remote transducer $(n=1)$. Data in the remotely measured $V_{A R}(t)$ waveform can be ascribed to the continuously changing interrogated point from which it originates by integrating the measured whole body velocity $\dot{x}$. However, the remote measurement provides only instantaneous data for any given 'point' on the target unless $\dot{x}$ is extremely small.

Greatest distortion of $V_{A R}(t)$ from the sinusoidal form of $V_{A F}(t)$ is observed when the remote measurement interrogates points where the spatial gradient of the modeshape has greatest magnitude, near the nodes of the modeshape. The distorting effect at any value of $x$ increases with increasing $\dot{x}$. Both $V_{A F}(t)$ and $V_{A R}(t)$ appear zero simultaneously in Figure 2, since $\sin (\omega t)=0$ is true at all points on the plate simultaneously, regardless of the measurement perspective. The distortion is not harmonic, however, as will be discussed at relevant points later in this paper.

\subsubsection{Quantification of distortion in $V_{A R}(t)$}

The distorting effect can be quantified in the time domain by locating the local maxima and minima $t_{F}(p)$ and $t_{R}(p)$ of $V_{A F}(t)$ and $V_{A R}(t)$ respectively, where $p=1,2,3 \ldots$ identifies each maxima or minima. From equation (1), the local maxima and minima of $V_{A F}(t)$ occur when $\omega \cos (\omega t)=0$. By differentiating equation (2) for the case where $x_{A}=l / n$ and the target has constant translational velocity, $\dot{x}$, the local maxima and minima of $V_{A R}(t)$ are given by solutions to the equation : 


$$
\frac{\omega}{z}=\frac{\tan (\omega t)}{\tan (z t)}
$$

where $z=(n \pi \dot{x}) / l$ and can be considered as the temporal frequency of the moving modeshape when observed remotely. Equation (3) cannot be solved readily, but indicates the influence of the ratio $\omega / z$ on the degree of distortion. This can be quantified if the local maxima and minima, $t_{R}(p)$, are located numerically and compared to the readily predictable local maxima and minima $t_{F}(p)$. Since $V_{A F}(t)$ and $V_{A R}(t)$ are zero simultaneously, the distortion in time exhibited by each maxima or minima of a remotely measured waveform, $D(p)$, can be expressed as a fraction of the time between successive zeroes thus :

$$
D(p)=\frac{t_{R}(p)-t_{F}(p)}{\pi / \omega}
$$

$D(p)$ is plotted across the modeshape from $x=0$ to $x=l / n$ in Figure 3 for the case where $x_{A}=l / n$. Frequency ratios $\omega / z=100$ and $\omega / z=10$ are used to represent upper and lower regions of the typical measurement range respectively, showing the distortion reduction as $\omega / z$ increases. With reference to Figure 3 , a single remote measurement on a target undergoing whole body translation will exhibit increasing distortion as the interrogated point approaches a node. In this case, the distortion will cause $t_{R}(p)$ to lag $t_{F}(p)$ as the interrogated point approaches an antinode, whilst $t_{R}(p)$ leads $t_{F}(p)$ as the interrogated point moves away from an antinode towards a node. The shape of the curves shown in Figure 3 also illustrates how a single remote measurement will contain appreciable distortion over a wide range of values of $\omega / z$ unless the measurement is confined to the region immediately around an antinode. 
In measurements where $\dot{x}$ is a function of time, the distortion, which is dependent upon both $z(t)$ and the spatial gradient of the modeshape in the instantaneously probed region, can be calculated for a given measurement using the same numerical method.

\subsection{FREQUENCY DOMAIN CHARACTERISTICS}

Equation (2) suggests that, in the case of constant whole body target translation velocity, the response spectrum of $V_{A R}(t)$ will contain peaks at angular frequencies $\omega \pm z$. In practice, however, only data captured from points on the target near to the main point of interest are useful since excessive amplitude modulation and distortion in the time domain would otherwise be encountered, as discussed above. This implies that only a fraction of one spatial period of the modeshape will in general be included in the measurement, which constitutes insufficient data length to resolve separate spectral peaks at angular frequencies $\omega \pm z$. Under these conditions, response spectra measured remotely from targets translating with either constant or time varying $\dot{x}$ are alike, since both are dominated by short data length characteristics resulting in poor frequency resolution and broad spectral peaks.

A single remote measurement from points on a 'long' target covering several spatial periods of the modeshape will generally not suffer this data length problem and, in the case of constant $\dot{x}$, spectral peaks are observed at frequencies $(\omega \pm z) / 2 \pi$ with no peak at frequency $\omega / 2 \pi$. A simulated measurement covering several spatial periods of the modeshape is shown in Figure 4a, where $\omega=300 \mathrm{rads}^{-1}$ and $z=30 \mathrm{rads}^{-1}$ and the peak amplitudes are normalised by the amplitude of the single peak which would occur in the equivalent spectrum from a fixed transducer. (This normalisation will be used for 
all subsequent spectra of simulated data). For cases in which $\dot{x}$ is a function of time and the direction of motion does not reverse, $z$ is effectively frequency modulated by the changing whole body target velocity. The resulting response spectrum thus displays a pair of broad bands at frequencies $(\omega \pm z(t)) / 2 \pi$, where the band width corresponds to the values of $z$ encountered during the measurement. This is observable in Figure $4 \mathrm{~b}$ where the normalised spectrum is shown for simulated measurement data captured over several spatial periods of the modeshape when $z$ increases linearly with time from zero to $80 \mathrm{rads}^{-1}(12.7 \mathrm{~Hz})$ during the measurement.

Harmonic whole body translation of the target is an important special case of $\dot{x}$ as a function of time, in which the instantaneously probed region on the target does not become progressively further away from the initially probed region. In this case sufficient data can be collected to produce reasonably detailed response spectra pertaining to localised regions of the target. For sinusoidal whole body translation at angular frequency $\Omega$, these spectra are derived by means of Bessel functions and consequently contain the frequencies $\omega / 2 \pi$ and $(\omega \pm m \Omega) / 2 \pi$ where $m$ is an integer. Since the data corresponds to localised regions on the target, broadening of the $(\omega \pm m \Omega) / 2 \pi$ spectral peaks is dependent upon the spatial gradient of the modeshape in the measurement region as expected from the earlier discussion of distortion. This effect is demonstrated in Figure 5 which shows two normalised response spectra measured remotely from a simulated target undergoing harmonic whole body translation. In Figure 5a, the point of interest on the target was near an antinode, whilst Figure $5 \mathrm{~b}$ pertains to a point on the target where the spatial gradient of the modeshape is larger and exhibits correspondingly broader $(\omega \pm m \Omega) / 2 \pi$ spectral peaks. The main 
peak amplitude in the remote measurements is shown to be marginally lower than that from a fixed transducer.

\subsection{A PRACTICAL EXAMPLE OF A SINGLE REMOTE VIBRATION RESPONSE}

\section{MEASUREMENT}

A standing wave is established on the uppermost, or 'crown', surface of a hollow golf club head during impact with a golf ball. This vibration persists for approximately $3 \mathrm{~ms}$ and is of interest to club designers since, as demonstrated in a previous study [3], it contributes to the sound of the impact and hence to the player's perception of the 'feel' of the golf club. The surface vibration can be detected remotely during and immediately after the impact using a laser vibrometer positioned vertically above the crown. Remote measurements are advantageous in this situation as the crown mass is approximately $30 \mathrm{~g}$ and its vibration characteristics would be altered by addition of even a small contacting transducer.

An example measurement taken from a single remote transducer over the time in which the club head translates completely past the probe laser beam is shown in Figure 6. The measurement has a low frequency component due to rotation of the whole club head and a higher frequency component due to oscillation of the crown surface. The amplitude of the measured waveform reduces towards the end of the data length due to the combined effects of vibration decay and increasing proximity of the instantaneously probed region to the edge of the crown, which is nodal. Moderate distortion of the waveform, of magnitude $\approx 12 \%$, is expected near the nodes since $\omega / z \approx 33$. 
According to data gathered in [3], the impact excites more than one mode of vibration of the crown at closely spaced frequencies in the range $5-9 \mathrm{kHz}$. Figure 6 shows the time history of the impact and Figure 7, the corresponding spectrum, shows broad peaks in the frequency range of interest that are not in agreement with data in [3]. The ability to investigate detailed response from a single remote measurement is limited by waveform distortion and insufficient data length pertaining to any single, fixed point on the surface. The displacement compensation technique substantially improves the quality of data obtained from remote measurements on targets undergoing whole body translation and is the subject of what follows in this paper. Subsequent re-analysis of the golf club crown vibration emphasises how only qualitative vibration characteristics can be ascertained from a single remote measurement.

\section{A DOUBLE REMOTE MEASUREMENT OF STEADY-STATE VIBRATION ON A TRANSLATING BODY}

\subsection{DISPLACEMENT COMPENSATION}

Waveform distortion and amplitude modulation inherent in a single remote vibration measurement on a target undergoing whole body motion can be overcome to a great extent through use of a second simultaneous remote measurement, at a point $B_{R}$, made along a measurement axis parallel to the first remote measurement at $A_{R}$. Combining data from both remote transducers allows close estimation of the vibration time history at point $A_{F}$ on the target over the measurement duration, which is taken to be the time interval in which point $A_{F}$ moves between the regions probed by remote transducers located at points $A_{R}$ and $B_{R}$. If point $A_{F}$ lies distances $x_{A}$ from an arbitrary reference point on the target (in this case the target edge) and $c$ from point $B_{F}$, as shown in 
Figure 8, then a weighted sum of data from remote transducer measurements at $A_{R}$ and $B_{R}$ can be used to produce a close estimate of $V_{A F}(t)$. For digitally sampled measurements, data from the $i$ th sample is combined in this displacement compensation technique according to :

$$
V_{A C}(i \Delta t)=[1-\alpha(i \Delta t)] V_{A R}(i \Delta t)+\alpha(i \Delta t) V_{B R}(i \Delta t)
$$

where $V_{A C}$ is the displacement compensated surface vibration velocity at point $A_{F}$ and $\Delta t$ is the time between samples. $\alpha(i \Delta t)$ describes the position of $A_{F}$ between the two probed regions and is defined by $\alpha(i \Delta t)=\frac{1}{c}\left(\int_{0}^{i \Delta t} \dot{x} d t\right)$, where $0 \leq \alpha(i \Delta t) \leq 1$, determining the relative weights of the measurements from $A_{R}$ and $B_{R}$ in the combined measurement for any $t$. Figure 9 shows the displacement compensated waveform, $V_{A C}(t)$, using equation (5) for the case where $\omega / z=100\left(c=0.3 \mathrm{l} / n\right.$ and $\left.x_{A}=0.5 \mathrm{l} / \mathrm{n}\right)$. The genuine vibration, $V_{A F}(t)$, at point $A_{F}$, given by equation (1), is also plotted on Figure 9 to indicate how $V_{A C}(t)$ differs only by a slight amplitude modulation. This close estimate of $V_{A F}(t)$, produced from remote measurements, exists for the time in which point $A_{F}$ moves between points $A_{R}$ and $B_{R}$, yielding substantially more temporal data from point $A_{F}$ than the instantaneous point measurement provided by a single remote transducer.

\subsection{TIME DOMAIN CHARACTERISTICS}

The exact extent to which the waveform distortion and amplitude modulation of a single remote measurement are eliminated using displacement compensation is dependent upon the chosen measurement parameters, $x_{A}$ and $c$. In particular, the amplitude error varies with the value of $\alpha(i \Delta t)$, as shown in Figure 9. The maximum amplitude error, $e_{M A X}$, in a given displacement compensated waveform is defined as : 


$$
e_{M A X}=\operatorname{MAX}\left[\left(\hat{V}_{A C}-\hat{V}_{A F}\right) / \hat{V}_{A F}\right]
$$

$e_{\text {MAX }}$ is defined for all displacement compensated measurements except for the trivial case where the point of interest on the target is a node. $e_{M A X}$ increases principally with increasing $c$ and, to a lesser extent, increasing proximity of $A_{R}$ or $B_{R}$ to a node. It is, therefore, important to choose values of $c$ and $x_{A}$ to suit both the point of interest and the mode of interest on the target body. The maximum useful separation of $A_{R}$ and $B_{R}$ is half of one spatial period of the mode of interest since the maximum amplitude error reaches $100 \%$ at this separation if, as a worst case example, the point of interest on the target is an antinode. In general, however, $e_{M A X}$ in the displacement compensated waveform is significantly smaller than the maximum amplitude error in a single remote measurement made under the same whole body target translation conditions. This is illustrated in Figure $10\left(x_{A}=0.4 l / n\right.$ and $\left.c=0.2 l / n\right)$, where the amplitude error for a single remote measurement is shown as a function of time with its displacement compensated equivalent, $V_{A C}(t) . V_{A C}(t)$ exhibits relatively minor amplitude error and thus provides a much better estimate of the fixed measurement $V_{A F}(t)$ over the measurement period than the single remote measurement.

The displacement compensated remote measurement is also a significant improvement on the single remote measurement in terms of the distortion of the waveform. The criterion introduced in Section 3.2 to quantify the waveform distortion is equally applicable to the displacement compensated measurement. Figure 11 shows the distortion encountered in two example compensated remote measurements for constant whole body target translation velocity when $\omega / z=10$, with the equivalent single remote measurement distortion, $D_{S}$, shown dotted. The two compensated 
measurements have $c=0.2 l / n$ and $c=0.4 l / n$ respectively and give distortion, $D_{C}$, calculated using equation (4). It can be seen that the lead-lag sense of the distortion is reversed in the compensated measurements and that the maximum distortion is less than from the equivalent single measurement, with least distortion occurring, not surprisingly, when the separation of the probed regions, $c$, is small. As with the single remote measurement, the level of distortion decreases with increasing $\omega / z$ and is, in addition, negligible for measurements in which $\omega / z$ is of the order of 100 or greater. This result indicates that the displacement compensated measurement, from a much wider range of measurement conditions, has minimal distortion and amplitude error when compared to the equivalent single remote measurement. Whilst this discussion has focused on constant $\dot{x}$ for clarity, this improvement in the data quality is also found when $\dot{x}$ is a function of time.

\subsection{FREQUENCY DOMAIN CHARACTERISTICS}

The displacement compensation technique does not extend the overall length of data captured from the translating target, hence frequency resolution is similar to that obtained from single remote measurements. However, in creating a waveform which closely approximates the genuine vibration at a fixed point over an interval, the displacement compensated data pertains to a considerably more localised region of the target surface than in the single remote measurement and, being virtually free of whole body target displacement effects, yields more representative frequency response information. This is discussed further in Section 4.4. 
When acquiring data, it is important to ensure that sample rate and anti-aliasing filters are set to allow for broadening of the original single remote measurements in the manner shown, for example, in Figure $4 b$.

The most obvious improvement on the single remote measurement in the frequency domain occurs in the case of harmonic whole body motion of the target. Here the response spectrum from displacement compensated data exhibits a peak at the vibration frequency $\omega / 2 \pi$ but no peaks at $(\omega \pm \Omega) / 2 \pi$, unlike the single remote measurement. This is confirmed by comparison of the single remote measurement spectrum shown earlier in Figure 5a with the equivalent displacement compensated spectrum, shown in Figure 12. This comparison additionally shows that sidebands at $(\omega \pm 2 \Omega) / 2 \pi$ are retained in the spectrum of the compensated data. In a compensated waveform the amplitude error is zero when $\alpha(i \Delta t)=0,1$ and the maximum error occurs at an intermediate value of $\alpha(i \Delta t)$. A single period of whole body harmonic target displacement involves one cycle of $\alpha(i \Delta t)$ (from 0 to 1 to 0 ) and thus two cycles of the amplitude error, such that the fundamental frequency of the amplitude error in the compensated waveform is $2 \Omega / 2 \pi$, resulting in the sidebands seen in Figure 12 .

\subsection{A PRACTICAL REMOTE MEASUREMENT PROBLEM}

The golf club crown vibration response data discussed in Section 3.4 can be improved considerably by application of the displacement compensation technique. Crown surface velocity data was captured over a series of impacts using a laser Doppler vibrometer positioned at nine remote locations, separated by $1 \mathrm{~cm}$, in a straight line vertically above the line of motion of the club head. This measurement separation is 
less than half of one spatial period of the modes of interest. (In this case the nine data sets are not simultaneous and compensation relies on the good repeatability of the impact conditions). The golf club translates a distance of approximately $10 \mathrm{~cm}$ in the period of interest, thus data from several of the remote measurement pairs is used to estimate the vibration response at a given fixed point on the crown surface. The practical arrangement is illustrated in Figure 13 where it can be seen that, for example, data from points $A_{R}$ to $G_{R}$ will at some time during the measurement period pertain to the vibration response at point $G_{F}$ on the target. The exact contribution of each remote measurement is determined by the separation of the probed regions and the whole body target displacement, $x(t)$, derived independently from an accelerometer attached to a convenient position elsewhere on the club head.

Application of the displacement compensation technique produces vibration response data for each of the points $D_{F}-J_{F}$ and also for any intermediate point of interest on the target not explicitly defined as a measurement location $D_{F}-J_{F}$. Approximately $3 \mathrm{~ms}$ of vibration response data is available for a given fixed point on the target, which is substantially more data than can be obtained for a single fixed point from a single remote measurement and is obtained considerably more conveniently than by the attachment of a contacting transducer at several locations on the surface. In this study, the low frequency part of the compensated signal, which corresponds to rotation of the whole club head, is of little importance and can be removed using a high pass filter with $2 \mathrm{kHz}$ cut-off.

The compensated data thus obtained for point $H_{F}$ is shown in Figure 14. The amplitude modulation is due to a beat between two principal vibration modes excited at similar 
frequencies by the impact. The response spectrum of this measurement, Figure 15 , shows the two beating frequencies to be $5700 \mathrm{~Hz}$ and $6250 \mathrm{~Hz}$ which agree very closely with the experimental and computational modal analysis of the golf club head presented in [3]. Similarly, smaller peaks at 5.0, 6.8, 7.4 and $8.2 \mathrm{kHz}$ present in Figure 15 are also in agreement with those reported in [3]. This quality of information was not found in the single remote measurement data, preventing any useful theoretical experimental comparison and this underlines the importance of displacement compensation.

\section{APPLICATION OF DISPLACEMENT COMPENSATION TO}

\section{IMPACT TRANSIENTS}

\subsection{MEASUREMENT SITUATION}

Detailed analysis of bodies subjected to impact necessitates the measurement of transient effects such as wave motion in a body. In an impact, a stress pulse will propagate away from the contact site through both colliding bodies. This pulse motion can be observed from a series of point measurements taken from transducers arranged along the body in the direction of propagation of the pulse. Most impacts involve large scale motion of at least one of the impacting bodies either before or after the collision, thus signal retrieval by electrical connection is difficult and damage may occur to a transducer attached to the moving body. Also, when the impacting bodies are of low mass, the additional mass of a transducer could significantly affect the system under investigation. It is therefore desirable to measure wave motion in impacted bodies by remote means. 
The laser Doppler vibrometer is an appropriate instrument for remote measurement of transient effects in impacted bodies. This section describes how the vibrometer can be employed successfully in the measurement of a pulse propagating through a moving body by extending the displacement compensation technique introduced in Section 4 . A pulse of arbitrary and continuously evolving shape is considered propagating through a body translating under large and variable acceleration, caused by an impact. The method is then applied to the analysis of transient deformation in an impacted golf ball to illustrate how the displacement compensation technique is able to estimate closely the vibration of a single fixed point on a body during impact.

\subsection{CHARACTERISTICS OF THE REMOTE MEASUREMENT}

An impact will generally cause a pulse to propagate through a body in the direction of the impact and the whole body will also accelerate in that direction. For a nondispersive pulse, the velocity of the pulse relative to a transducer fixed to the body is simply equal to the constant speed, $\dot{x}_{p}$, of pulse propagation in the impact direction. For a dispersive pulse, the 'group velocity' can be considered by equivalent means. Thus, for a pulse of length $\lambda$, a transducer fixed to the body will record a pulse of duration $T_{F}=\lambda / \dot{x}_{P}$. However, the velocity of the pulse relative to a remote transducer is equal to the sum of the propagation speed and the whole body velocity. Therefore, the remote transducer records a pulse of duration $T_{R}=\lambda\left(\dot{x}_{p}+\dot{x}\right)$ which is shorter than $T_{F}$. The remote measurement thus gives a waveform which contains amplitude information equivalent to a fixed transducer but which is distorted in time according to the motion of the body. For data captured digitally at the same sample 
rate, $f_{S}$, from fixed and remote transducers, fewer samples will be in the remotely measured pulse and the effective sample rate is lower than $f_{S}$ by a factor $T_{R} / T_{F}$. This must be recognised in making the appropriate choice of anti-aliasing filter for the measurement conditions.

\subsection{ADAPTATION OF THE DISPLACEMENT COMPENSATION TECHNIQUE}

A simulated, arbitrary, dispersive pulse on an impacted body measured from two remote locations by transducers, arranged as in Figure 8, is shown in Figure 16a. The remotely measured quantities are the surface velocities of the body perpendicular to the impact direction, $V_{A R}(t)$ and $V_{B R}(t)$, and these are plotted against the sample number of each digitally stored data point. The displacement compensation technique is applied in order to reconstruct a close approximation to the measurement which would be made by a transducer fixed to point $A_{F}$. However, the situation is complicated by pulse shape changes and variable distortion in time according to the changing translational velocity of the whole body.

In Figure 16a the two pulse measurements $V_{A R}(t)$ and $V_{B R}(t)$ appear separated in time by a number of samples $n_{C}$ which is a function of the velocity of propagation of the pulse, $\dot{x}_{P}$, and the translational velocity of the body, $\dot{x}$. Each pulse measurement is also divided into two intervals such that the pulse rises from zero to a maximum in the first interval and falls from maximum to zero in the second. Each interval is considered separately and in the first interval $V_{A R}(t)$ comprises $n_{A I}$ samples whereas $V_{B R}(t)$ comprises $n_{B I}$ samples. The pulse shape evolves continuously as it propagates across the body, thus for the $i$ th sample on $V_{A R}(t)$ a linear interpolation is proposed to identify the corresponding sample on $V_{B R}(t)$ such that: 


$$
j=\left(n_{B 1} / n_{A 1}\right) i+n_{C}
$$

A weighted sum of $V_{A R}(i \Delta t)$ and $V_{B R}(j \Delta t)$ is proposed to estimate the velocity at $A_{F}$ as it moves between measurement locations $A_{R}$ and $B_{R}$ :

$$
V_{A C}(k \Delta t)=[1-\alpha(k \Delta t)] V_{A R}(i \Delta t)+\alpha(k \Delta t) V_{B R}(j \Delta t)
$$

Equation (8) is a development of equation (5) and describes the general case of displacement compensation of a waveform from a double remote measurement. The essential difference from equation (5) is the presence of the term $k \Delta t$, which is necessary to correctly represent both the shape change of the pulse and the whole body velocity of the body. The physical significance of $k \Delta t$ can be described by considering Figure $16 \mathrm{~b}$, where the two remote measurements shown previously in Figure 16a are plotted against time and their respective fixed positions in space. Figure 16b shows geometrically the construction of a point on the compensated waveform using data from point $i \Delta t$ on the pulse measured at $A_{R}$ and the corresponding point $j \Delta t$ on the pulse measured at $B_{R}$. These points are shown connected by a straight line $I J$ in the $x-t$ plane and $k \Delta t$ is then defined by the intersection in the $x-t$ plane of line $I J$ and the whole body displacement curve $x(t)$ which represents the changing position of $A_{F}$. Geometrically, $k$ can be shown, from similar triangles on Figure $16 \mathrm{~b}$, to be :

$$
k=[(j-i) \alpha(k \Delta t)]+i
$$

In practice, the intersection relating $i, j$ and $k$ must be obtained for every $k$ in which $0 \leq \alpha(k \Delta t) \leq 1$ and can be found in a number of ways.

In the most direct method, $k$ is selected as the known variable, which explicitly defines the value of $\alpha(k \Delta t)$ and allows the corresponding $i$ at the intersection to be found directly if equations (7) and (9) are combined to form the following equation : 


$$
i=\frac{k-n_{C} \alpha(k \Delta t)}{\left[\left(\frac{n_{B 1}}{n_{A 1}}-1\right) \alpha(k \Delta t)+1\right]}
$$

The value of $i$ can be used to evaluate $j$ from equation (7) and $V_{A C}(k \Delta t)$ is then found from equation (8) for all $k$ in which $0 \leq \alpha(k \Delta t) \leq 1$. The discrete nature of the data implies the value of $i$ determined using equation (10) must be rounded to the nearest integer. This introduces an error of less than $\Delta t$, which is, in practice, very small for most digitally sampled data. However, rounding also introduces the possibility that an increment in $k$ may result in a rounded value of $\Delta i=0$, requiring repeated use of the same data, $V_{A R}(i \Delta t)$ and $V_{B R}(j \Delta t)$, in equation (8) which would create an undesirable 'flat spot' in the compensated waveform.

An alternative approach is to select $i$ as the known variable and to locate the intersection of lines $I J$ and $x(t)$ numerically. This produces the opposite effect in which an increment in $i$ can produce a rounded value of $\Delta k>1$, which creates a gap of one or more samples in the compensated waveform. Equation (9) shows that three effects determine the occurrence of gaps in the compensated waveform. Firstly, the value $n_{B I} / n_{A I}$ is usually greater than 1 due to spatial broadening of pulses. A large value of $n_{B 1} / n_{A l}$ implies a large shape change of the pulse between measurement points, potentially giving rise to $\Delta k>1$. Secondly, in the case of no pulse shape change, $k=\left[n_{C} \alpha(i \Delta t)\right]+i$, indicating that a large value of $n_{C}$, which would occur due to a high wave propagation speed or a large separation of the probed regions, can produce $\Delta k>1$. Thirdly, large $\Delta \alpha$ (whilst $\alpha$ remains in the range $0 \leq \alpha(i \Delta t) \leq 1$ ) would be produced by a high translational velocity of the body, again potentially giving $\Delta k>1$. 
Gaps can be closed smoothly by interpolating linearly across the gap, introducing a negligibly small error.

In practice, the required intersection between lines $I J$ and $x(t)$ can be found for each value of $i$ using an algorithm written into a computer program which firstly defines the line $I J$ in the $x$ - $t$ plane using the following equation :

$$
x_{I J}=\left(\frac{c}{j-i}\right)\left(\frac{t-i \Delta t}{\Delta t}\right)+x_{A}
$$

For every sample in $V_{A R}$, an array of $x_{I J}$ values is created from equation (11) as $t$ is incremented from $i \Delta t$ to $j \Delta t$. The time, $k \Delta t$, at which the intersection between this line and the whole body displacement $x(t)$ occurs is determined using a least squares error procedure. $\alpha(k \Delta t)$ can then be evaluated from $\alpha(k \Delta t)=\left(x_{k}-x_{A R}\right) / c$ to determine the relative contributions of $V_{A R}(i \Delta t)$ and $V_{B R}(j \Delta t)$ in equation (8). This is indicated by projection of the intersection point $R$ vertically upwards onto the straight line $V_{A R}(i \Delta t) V_{B R}(j \Delta t)$ at point $S$ in Figure $16 \mathrm{~b}$

When the displacement compensation process is complete, the compensated pulse has a number of samples equal to the number in the equivalent measurement made by a transducer fixed to the body. However this does not imply the effective sample rate of the compensated measurement is equal to $f_{S}$, since the data in $V_{A C}(t)$ is originally captured at an effectively lower sample rate and filtered accordingly.

\section{DISPLACEMENT COMPENSATION OF WAVEFORMS}




\subsection{MEASUREMENT SITUATION}

The golf ball is a lightweight viscoelastic sphere subjected to a high speed impact of approximately $450 \mu \mathrm{s}$ duration, causing it to be projected away from the impact site. During impact the ball is accelerated from rest to around $55 \mathrm{~ms}^{-1}$ and significant deformation of the ball occurs both in the direction of the impact force and perpendicular to it. Detailed remote measurements of golf ball deformation and forward motion during impact have been previously reported in [4]. Figure 17 shows a plan view of the measurement set-up employed in [4] to capture lateral deformation velocity data, $V_{A R}(t)$ and $V_{B R}(t)$, by remote means at points on the equator of a golf ball using two laser vibrometers. A third remote measurement at point $B_{R}{ }^{\prime}$, shown dotted, contains components of both lateral deformation velocity and forward velocity of the ball and is used in conjunction with $V_{B R}(t)$ to extract the forward velocity of the ball, $\dot{x}(t)$. The vibrometer measurements are not affected by the spherical shape of the golf ball or by the presence of dimples on the surface since the instrument is sensitive only to the velocity of the illuminated particles on the surface [5]. The measured lateral deformation velocities, $V_{A R}(t)$ and $V_{B R}(t)$, shown in Figure 18, are positive in the direction of the vibrometer in the first half of the impact, whilst in the second half of the impact the ball attempts to recover its original shape and the measured velocity is negative. The separation of $V_{A R}(t)$ and $V_{B R}(t)$ in time indicates the progression of the lateral deformation across the ball in the positive $x$-direction during the impact. The measurements also exhibit an amplitude change due to the variation in cross section of the ball and a shape change due to spatial broadening as the wave propagates through the viscoelastic ball material. In [4], forward velocity measurements indicated that in the first $150 \mu$ s of impact with a golf club the ball deforms whilst remaining stationary. 
After this time the whole ball accelerates forwards in a non-linear manner and translates a distance of $11 \mathrm{~mm}$ in the remaining $300 \mu \mathrm{s}$ of the impact. Lateral deformation velocity measurements made remotely using a laser vibrometer thus originate from instantaneously probed regions on the ball surface increasingly distant from $A_{F}$ and $B_{F}$.

\subsection{APPLICATION OF DISPLACEMENT COMPENSATION TO GOLF BALL DEFORMATION MEASUREMENTS}

The displacement compensation technique described in Section 5 is directly applicable to the golf ball lateral deformation measurement. $V_{A R}(t)$ and $V_{B R}(t)$ can be divided into compensating intervals defined by their maximum, minimum and zeroes and used, along with the forward displacement history of the golf ball, to calculate the compensated waveform in each interval and hence for the entire impact by application of equation (8). The compensated waveform, $V_{A C}(t)$, is also shown in Figure 18 for direct comparison with its uncompensated equivalent $V_{A R}(t)$. The comparison reveals how $V_{A C}(t)$ is very similar to $V_{A R}(t)$ in the first half of the impact as a consequence of the negligible forward displacement of the whole ball in this interval. Rapidly increasing forward displacement in the second half of the impact gives greater weight to $V_{B R}(t)$ in equation (8) and causes $V_{A C}(t)$ to resemble the $V_{B R}(t)$ measurement more closely. At time $400 \mu$ s into the impact the golf ball has translated a distance of $9 \mathrm{~mm}$, which is equal to the spacing of the regions probed by the two laser beams. $V_{A C}(t)$ should therefore be exactly equal to $V_{B R}(t)$ at this time and is seen to be the case in Figure 18 . This is basic evidence for the ability of the displacement compensation technique to produce a closer estimate of the transient deformation velocity at a point on an impacted body using data from two remote point measurements. Slight inaccuracies 
may remain but can be tolerated since this measurement on a lightweight body projected far from the impact site would be extremely difficult to make by any other means. Further information at times beyond $400 \mu \mathrm{s}$ is obtainable in this case by increasing the measurement spacing at the expense of a slightly reduced pulse shape change interpolation accuracy, or through introduction of a third measurement in the manner demonstrated with hollow golf club head data in Section 4.4. The measurement capability afforded by the displacement compensation technique in impact situations is important since it permits direct comparison of experimental data, obtained under difficult measurement conditions, with the behaviour of fixed points on moving bodies in theoretical studies of the impact, an example of which would be individual nodes in a finite element model.

\section{CONSIDERATION OF LASER SPECKLE EFFECTS}

When making remote measurements on a surface using the laser Doppler technique, consideration must be given to the speckle pattern which is produced whenever coherent light is scattered from a surface that is rough on the scale of the optical wavelength. In-plane translations of the target surface cause changes in the spatial characteristics of the speckles collected by the vibrometer's photodetector, producing amplitude and phase variation in the photodetector output which may, in some cases, be mis-interpreted as the genuine vibration [6].

The variation in the photodetector output caused by speckle effects is, however, significantly smaller than the variation caused by genuine vibration of the target unless the measured vibration amplitude is very low. In the measurements on a golf ball 
presented in this paper, the speckle noise amplitude will increase with increasing translational velocity of the ball during the impact. However, the level of noise in the uncompensated signal shown in Figure 18 does not increase noticeably during the impact, suggesting that the speckle noise amplitude is low relative to the vibration amplitudes encountered. This was confirmed in simple laboratory tests in which a falling golf ball passed through the probe laser beam.

For a target which undergoes harmonic translation at frequency $\Omega$, the periodic nature of the resulting speckle noise will produce peaks in the measured response spectrum at frequency $\Omega$ and higher order harmonics. These unwanted peaks are of small amplitude in the majority of cases but there is some potential for ambiguity if speckle noise harmonics extend into the frequency range of interest and, in particular, if there is a match between $\omega$ and an inferger multiple of $\Omega$. The presence of speckle effects must therefore be acknowledged when interpreting response spectra derived from remote measurements on targets which undergo harmonic translation.

In the displacement compensation of vibration data measured remotely from the club head and ball, translation of the target occurs only in one direction and the speckle pattern does not repeat. The speckle noise does not, therefore, produce peaks in the measured response spectrum and instead a slight increase in noise across a broad frequency range is encountered. The ability to identify resonant peaks in the spectrum is, therefore, largely unaffected by the speckle noise.

\section{CONCLUSION}


A measurement and analysis system has been introduced which permits close approximation of the vibration of fixed points on bodies undergoing whole body translation from data recorded using remote transducers. This paper has investigated the characteristics of a single remote measurement of steady state vibration on a translating body in the time and frequency domains. In the time domain, problems of waveform distortion and amplitude modulation are quantified whilst in the frequency domain, the presence of whole body target displacement induced harmonics are illustrated, together with the effects of short data length. A displacement compensation technique is introduced in which a second simultaneous remote measurement is used, along with the target's whole body displacement history, to derive a closer estimate of the measurement which would be made by a contacting transducer fixed to a point on the target surface. The improvement in data quality, relative to a single remote measurement, is quantified by reference to both simulated and actual experimental data and is apparent in both the time and frequency domains. The displacement compensation technique is also appropriate to impact studies and has been developed in this paper to investigate transient pulse propagation effects in impacted bodies remotely, thus permitting the close approximation of fixed point data which would otherwise be extremely difficult to obtain.

The capability to derive data effectively from a single point on a moving body using remote transducers in difficult measurement situations is a valuable experimental tool with applications in structural vibration analysis and beyond. As remote, non-contact measurement methods become increasingly popular, the work presented in this paper 
demonstrates the improvement in the quality of data which can be obtained using the displacement compensation technique.

\section{ACKNOWLEDGEMENTS}

This study was carried out as part of an Engineering and Physical Sciences Research Council grant investigating the design analysis of sculptured surface products. The authors would like to acknowledge the assistance of Dunlop Slazenger International Limited. 


\section{REFERENCES}

1. N.A.Halliwell 1993 Optical Methods in Engineering Metrology. (Ed: Williams,DC) Chapman \& Hall, London, UK, chapter 6. Laser Vibrometry.

2. A.B.Stanbridge and D.J.Ewins 1995 Proceedings of the ASME, Design Engineering Technical Conference, Boston, USA. Vol. 84-2. 1207-1213. Modal testing of rotating discs using a scanning LDV.

3. A.Hocknell, S.R.Mitchell, R.Jones and S.J.Rothberg 1997 Accepted for publication in Experimental Mechanics. Hollow golf club head modal characteristics : Determination and impact applications.

4. A.Hocknell, R.Jones and S.J.Rothberg 1996 Measurement Science and Technology 7(9), 1247-1254. Experimental analysis of impacts with large elastic deformation. Part 1 : Linear motion.

5. S.J.Rothberg and N.A.Halliwell 1995 Proceedings of the ASME - 15th International Conference on Vibration and Noise, Boston, USA. 1425-1433. Application of laser vibrometry to vibration measurement on rotating components.

6. S.J.Rothberg, J.R.Baker and N.A.Halliwell 1989 Journal of Sound and Vibration 135(3), 516-522. Laser vibrometry: Pseudo-vibrations. 
Remote vibration measurements : compensation of waveform distortion due to whole body translations

A.Hocknell, R.Jones \& S.J.Rothberg

Loughborough University

Figure 1 - Single remote measurement on a translating body, a) $t=0 \&$ b) $t>0$

Figure 2 - Simulated steady state vibration response measurement on a translating plate. First mode, $\omega=300 \mathrm{rads}^{-1}, l=0.2 \mathrm{~m}, u=1.9 \mathrm{~ms}^{-1}, x_{A}=0.18 \mathrm{~m}$.

$\left(---V_{A F}(t),-V_{A R}(t)\right)$

Figure 3 - Distortion in a single remote measurement

Figure 4a - Response spectrum for constant $\dot{x}$ translation of a 'long' target

Figure $4 \mathrm{~b}$ - Response spectrum for translation of a 'long' target when $\dot{x}$ is a function of time

Figure 5a - Harmonic whole body target translation : Response spectrum near antinode

Figure 5b - Harmonic whole body target translation : Response spectrum in a region of high spatial gradient of the modeshape

Figure 6 - Example vibrometer measurement from a golf club crown

Figure 7 - Response spectrum from a single remote measurement on a golf club crown

Figure 8 - A double remote measurement on a translating body 
Figure 9 - Displacement compensated waveform, $V_{A C}(t)$ and genuine vibration response $V_{A F}(t)$

Figure 10 - Amplitude errors in remote measurements

Figure 11 - Distortion in double remote measurements

Figure 12 - Response spectrum calculated from displacement compensated data in the case of harmonic whole body target translation

Figure 13 - Remote measurements used in the displacement compensation of data from a golf club head crown

Figure 14 - Displacement compensated data for point $H_{F}$

Figure 15 - Response spectrum of displacement compensated data for point $H_{F}$

Figure 16a - Example pulse measured by two remote transducers

Figure $16 \mathrm{~b}$ - Construction of the displacement compensated pulse

Figure 17 - Measurement setup to capture lateral deformation velocity data from a golf ball during impact

Figure 18 - Lateral deformation velocities, $V_{A R}(t)$ and $V_{B R}(t)$, measured remotely on a golf ball during impact and displacement compensated equivalent, $V_{A C}(t)$.

$\left(-\cdots-V_{A R}(t), \cdots-V_{B R}(t),-V_{A C}(t)\right)$ 

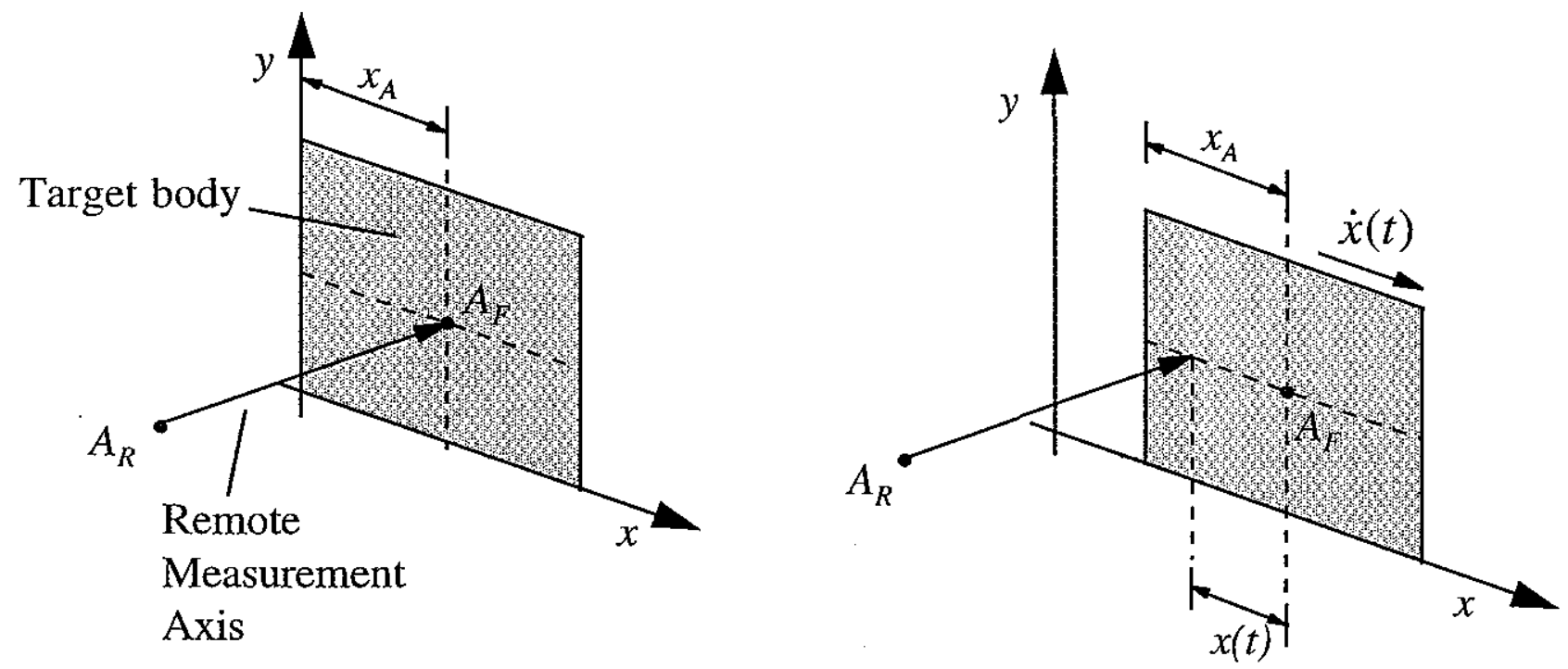

Figure 1

Remote vibration measurements : compensation of waveform

distortion due to whole body translations

A.Hocknell, R.Jones \& S.J.Rothberg

Loughborough University 


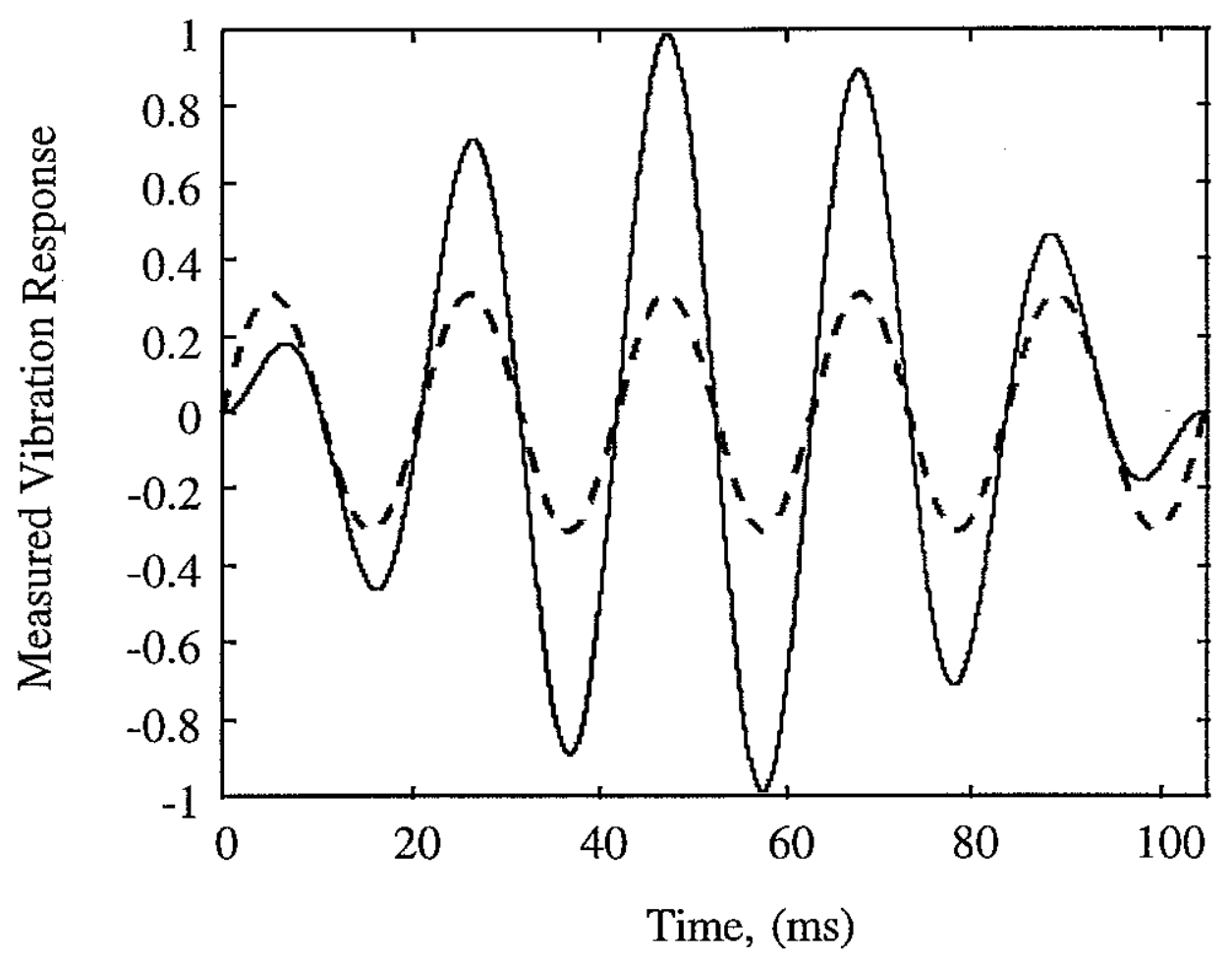

Figure 2

Remote vibration measurements : compensation of waveform distortion due to whole body translations

A.Hocknell, R.Jones \& S.J.Rothberg Loughborough University 


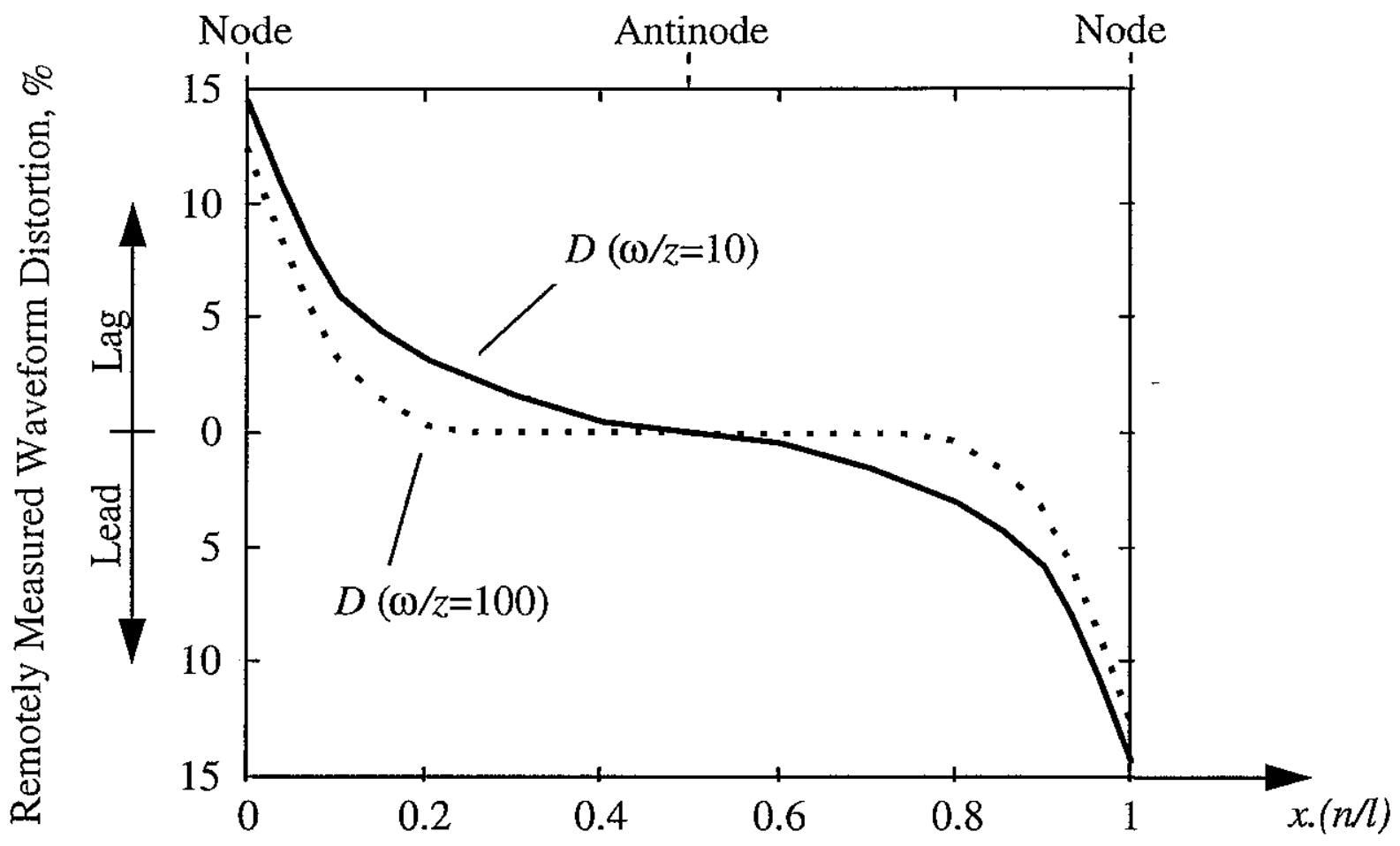

Figure 3

Remote vibration measurements : compensation of waveform distortion due to whole body translations

A.Hocknell, R.Jones \& S.J.Rothberg Loughborough University 


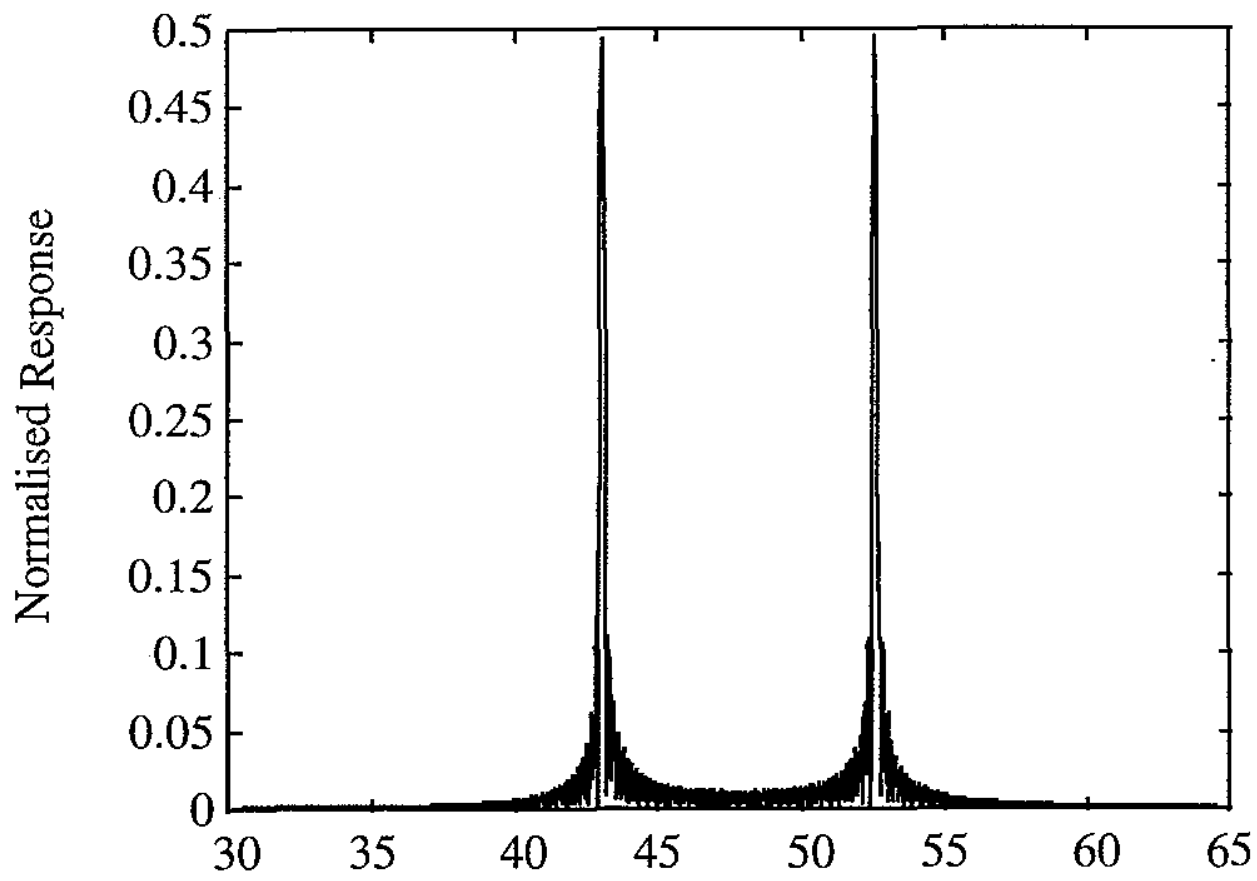

Frequency, (Hz)

Figure 4a

Remote vibration measurements : compensation of waveform distortion due to whole body translations

A.Hocknell, R.Jones \& S.J.Rothberg Loughborough University 


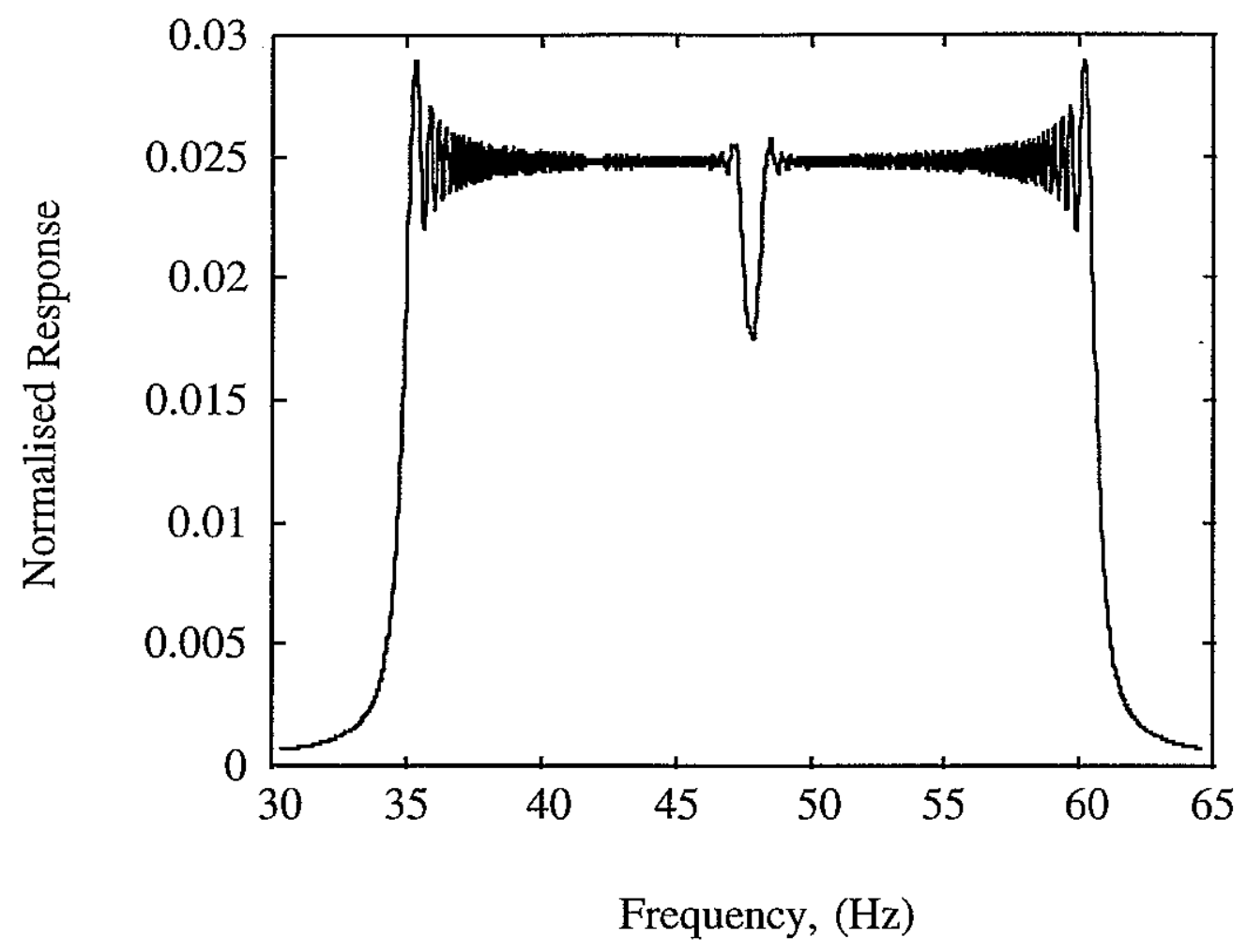

Figure $4 \mathrm{~b}$

Remote vibration measurements : compensation of waveform distortion due to whole body translations

A.Hocknell, R.Jones \& S.J.Rothberg Loughborough University 


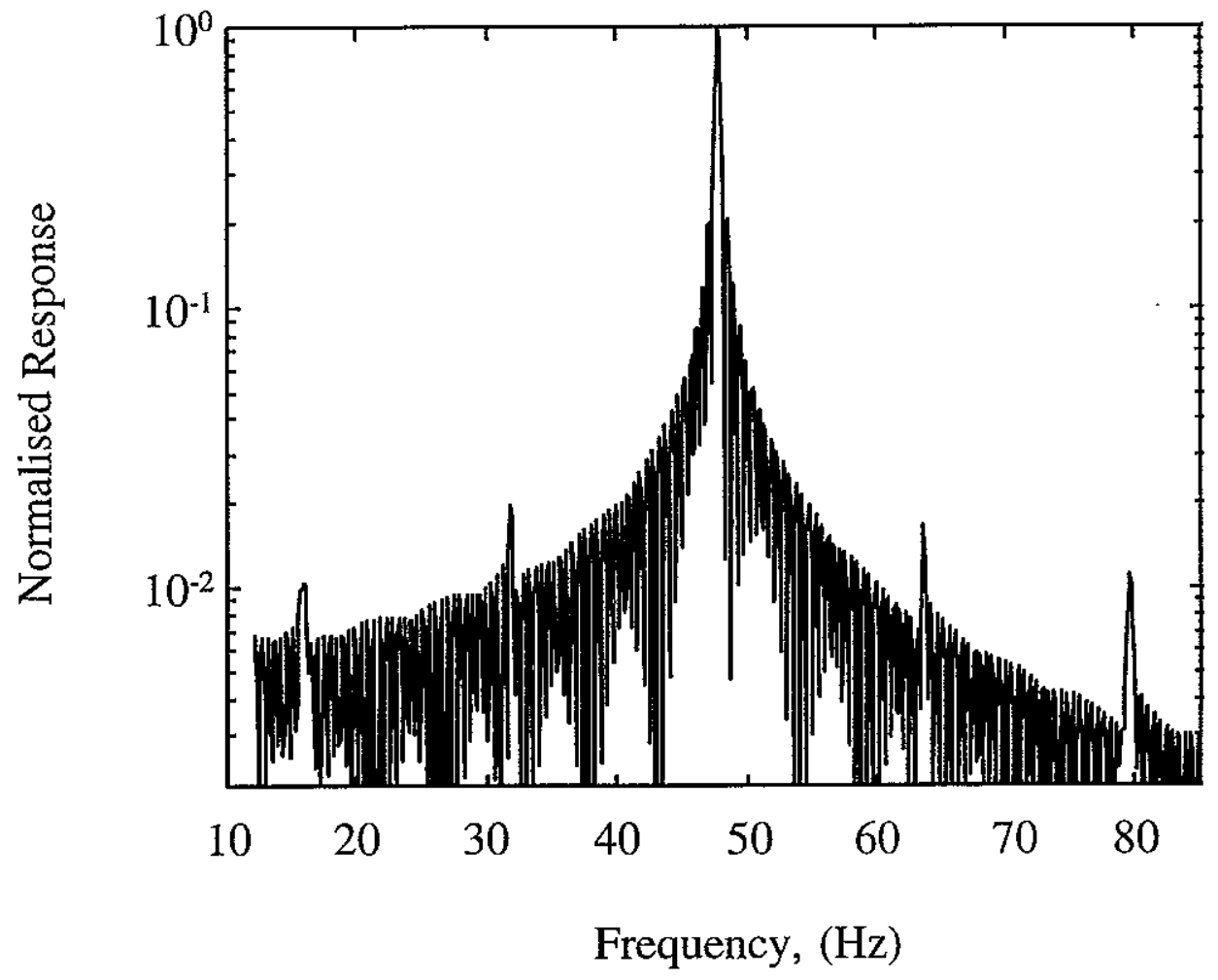

Figure 5a

Remote vibration measurements : compensation of waveform distortion due to whole body translations

A.Hocknell, R.Jones \& S.J.Rothberg Loughborough University 


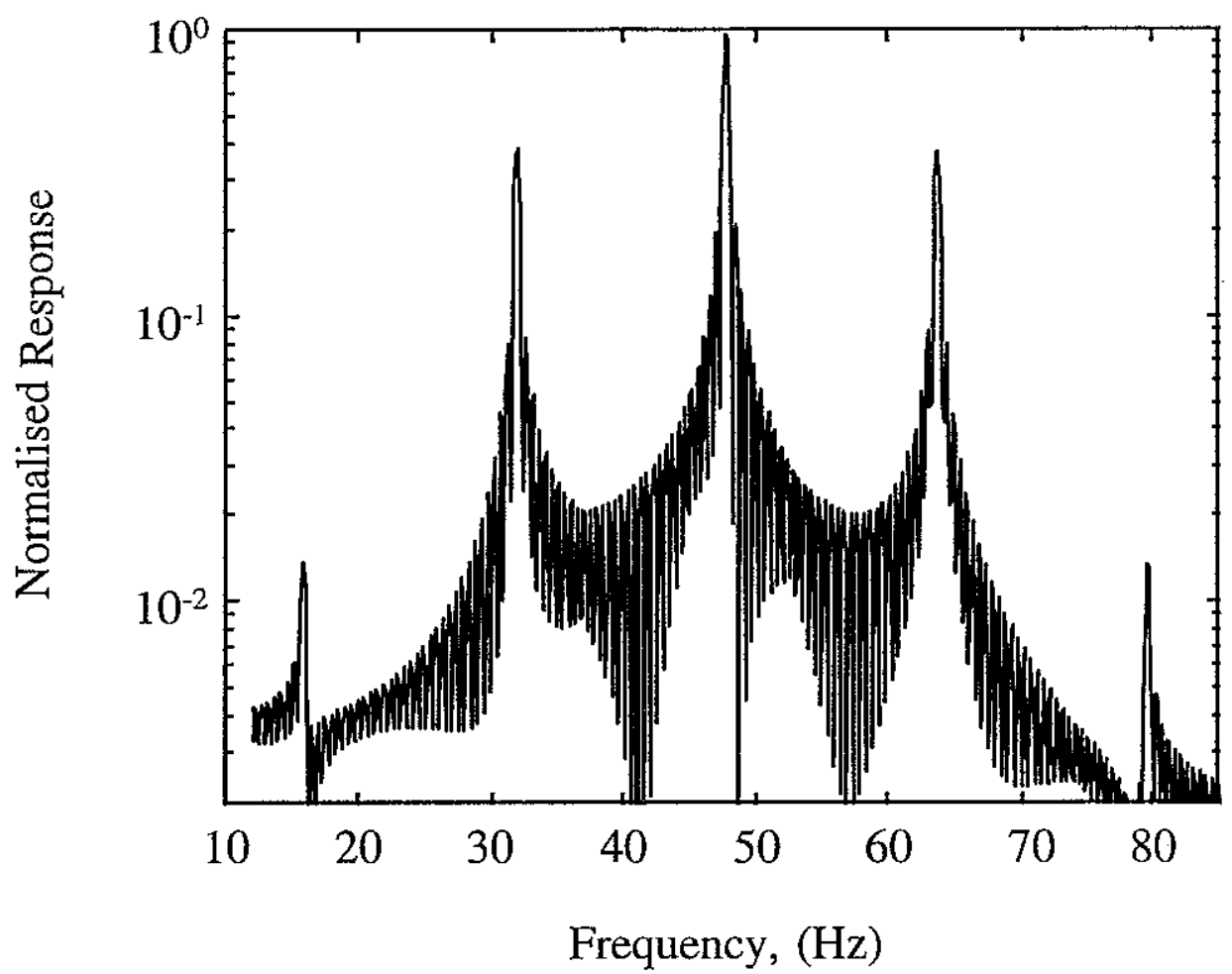

Figure $5 \mathrm{~b}$

Remote vibration measurements : compensation of waveform distortion due to whole body translations

A.Hocknell, R.Jones \& S.J.Rothberg Loughborough University 


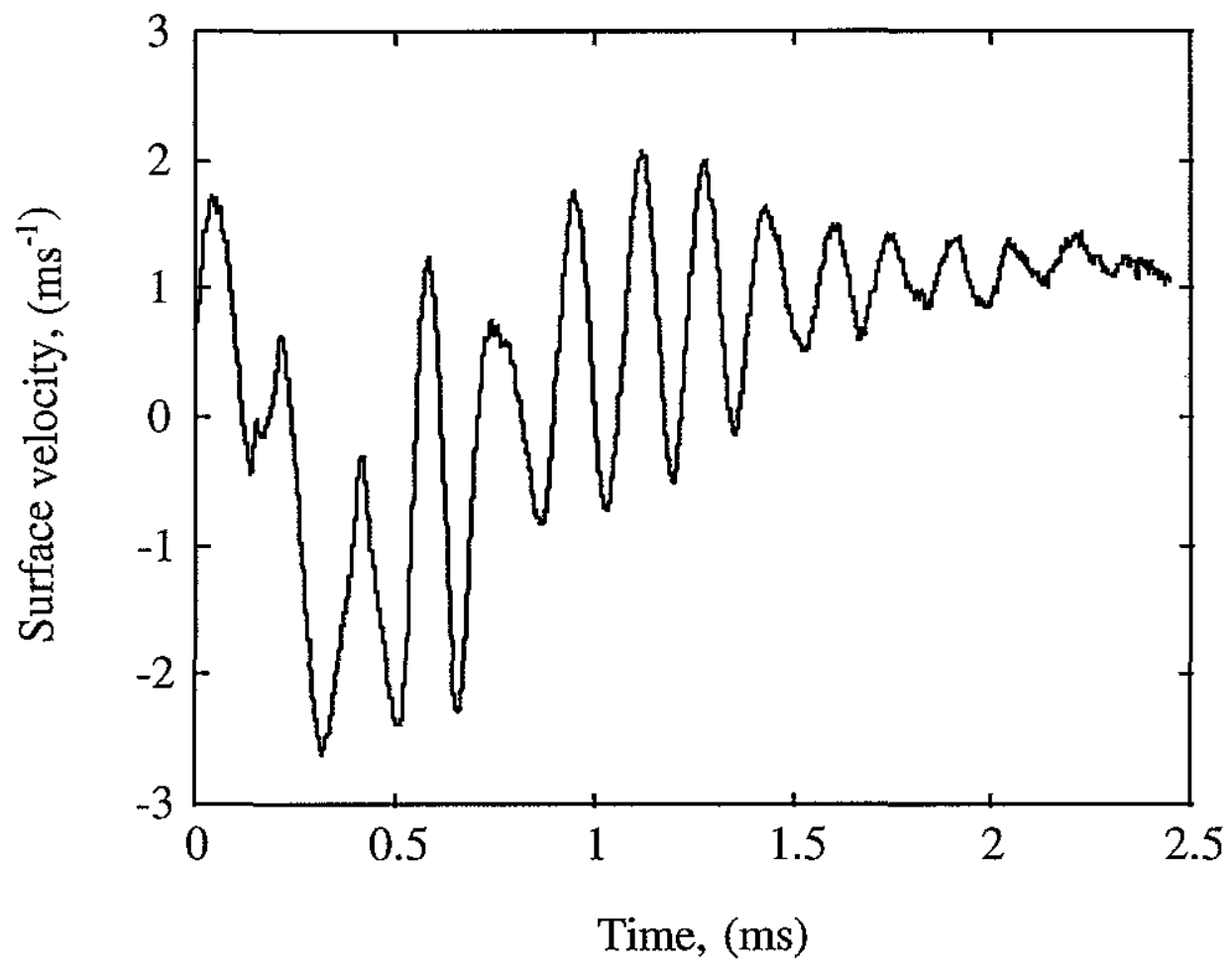

Figure 6

Remote vibration measurements : compensation of waveform distortion due to whole body translations

A.Hocknell, R.Jones \& S.J.Rothberg Loughborough University 


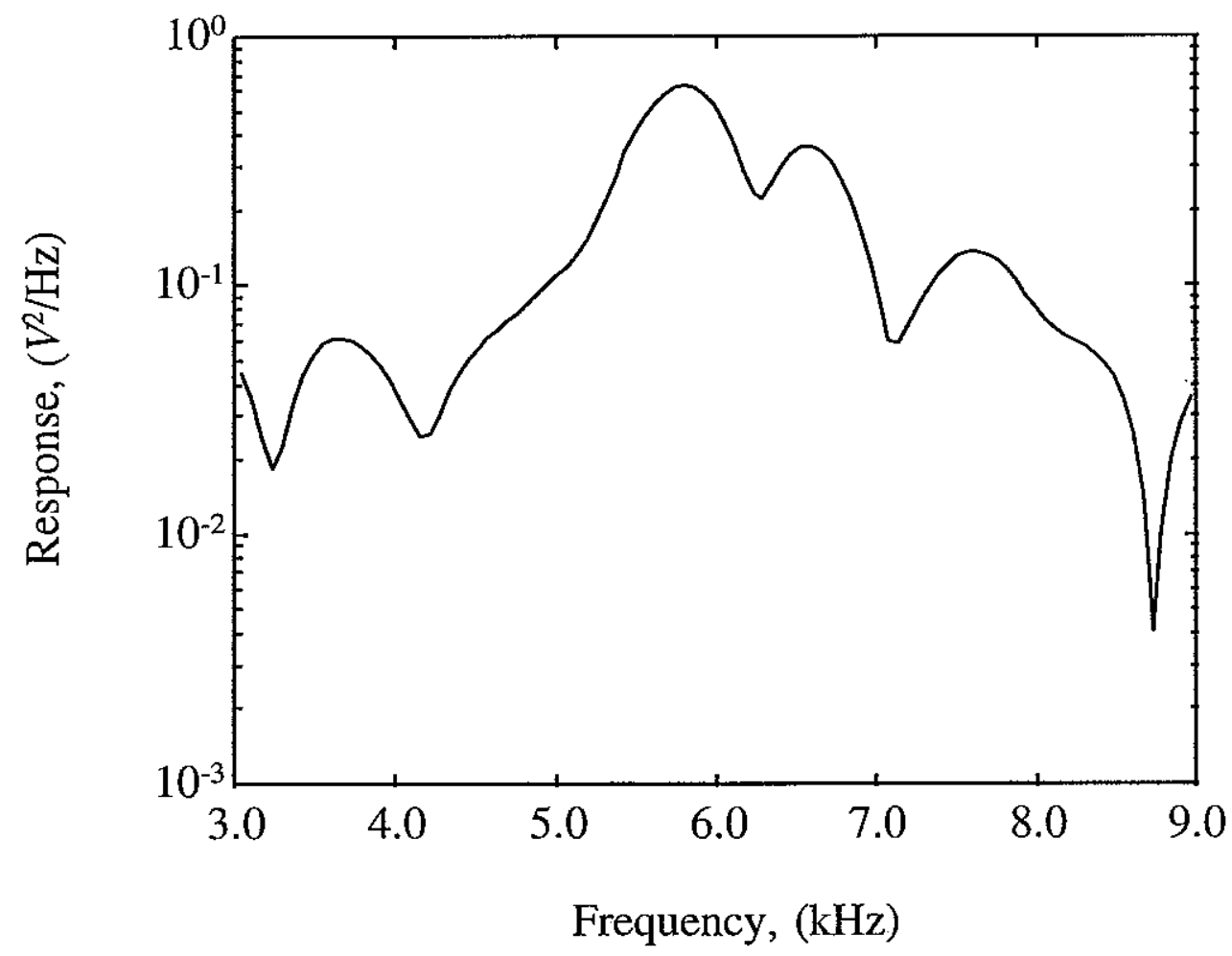

Figure 7

Remote vibration measurements : compensation of waveform distortion due to whole body translations

A.Hocknell, R.Jones \& S.J.Rothberg Loughborough University 


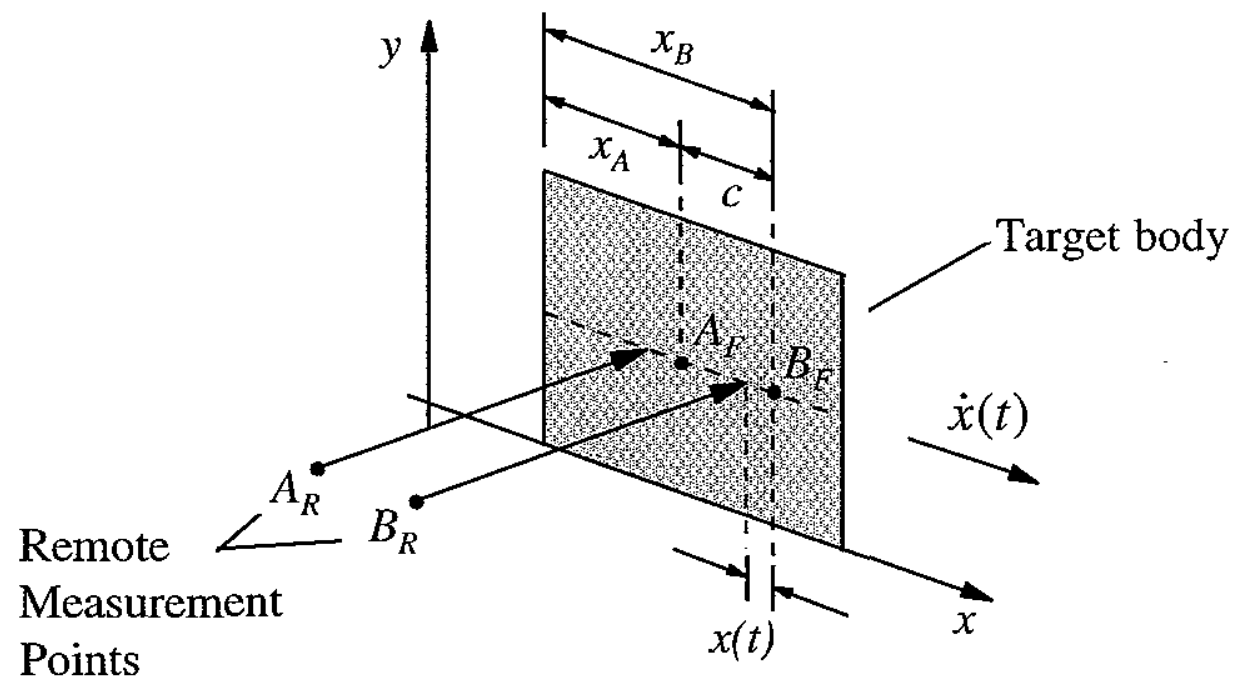

Figure 8

Remote vibration measurements : compensation of waveform distortion due to whole body translations

A.Hocknell, R.Jones \& S.J.Rothberg Loughborough University 
Slight amplitude modulation

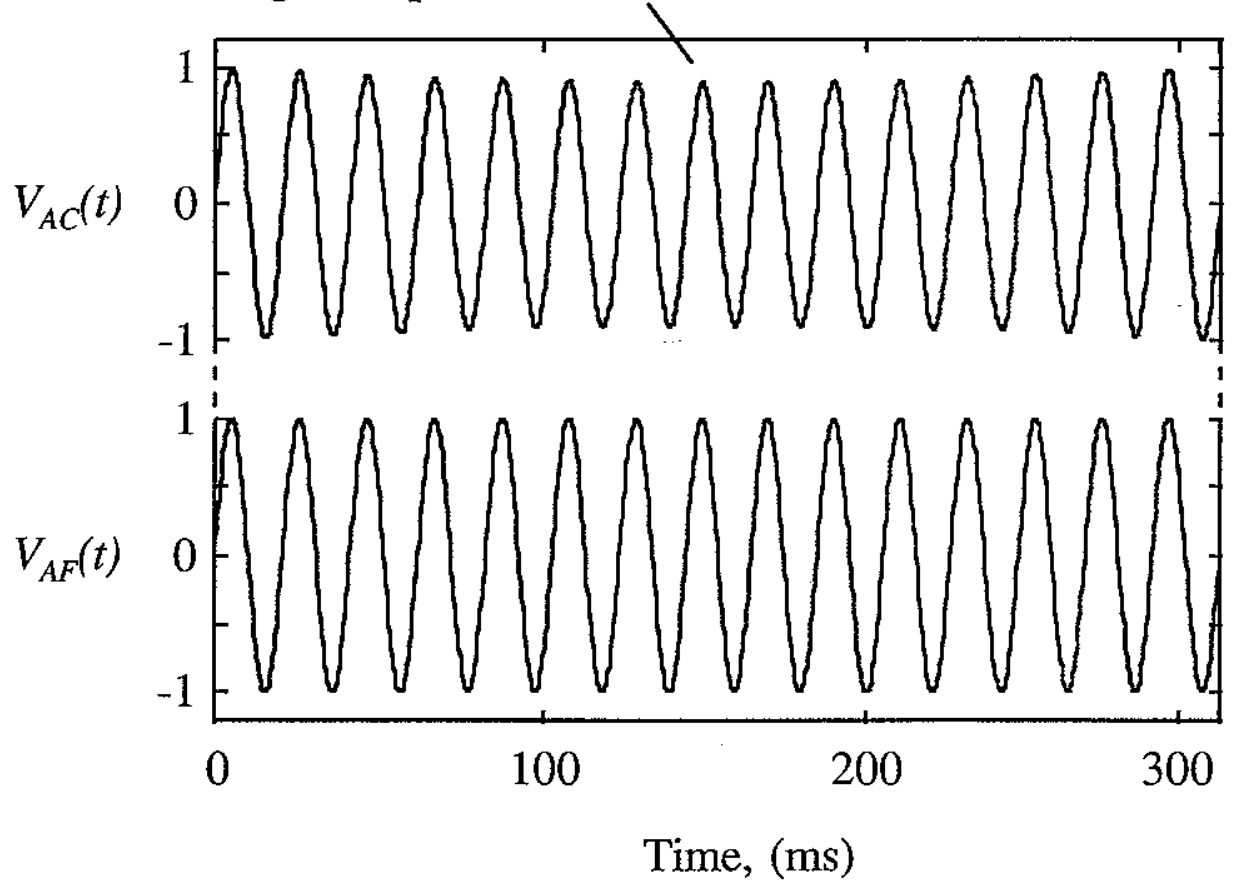

Figure 9

Remote vibration measurements : compensation of waveform distortion due to whole body translations

A.Hocknell, R.Jones \& S.J.Rothberg

Loughborough University 


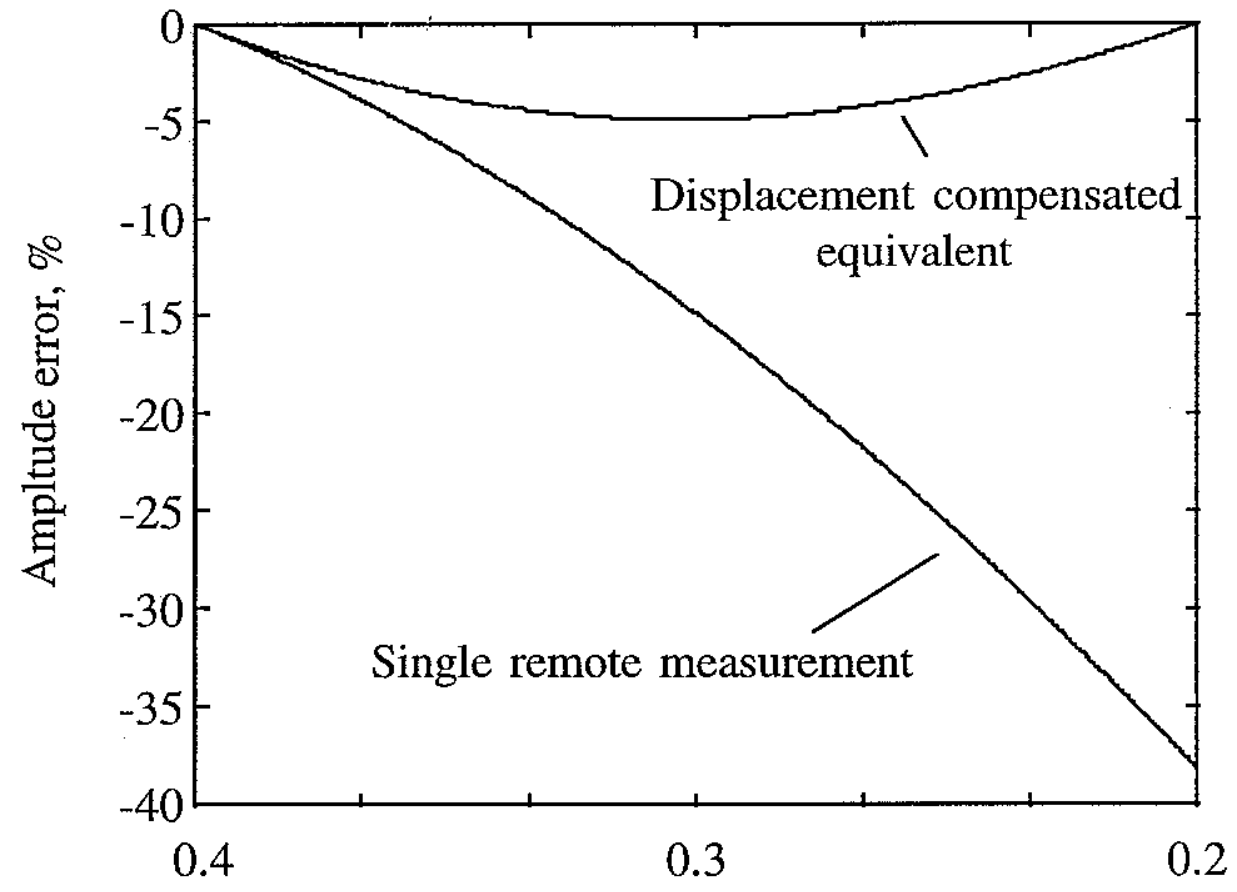

Point on the target interrogated by $A_{R}, x .(n /)$

\section{Figure 10}

Remote vibration measurements : compensation of waveform distortion due to whole body translations

A.Hocknell, R.Jones \& S.J.Rothberg

Loughborough University 


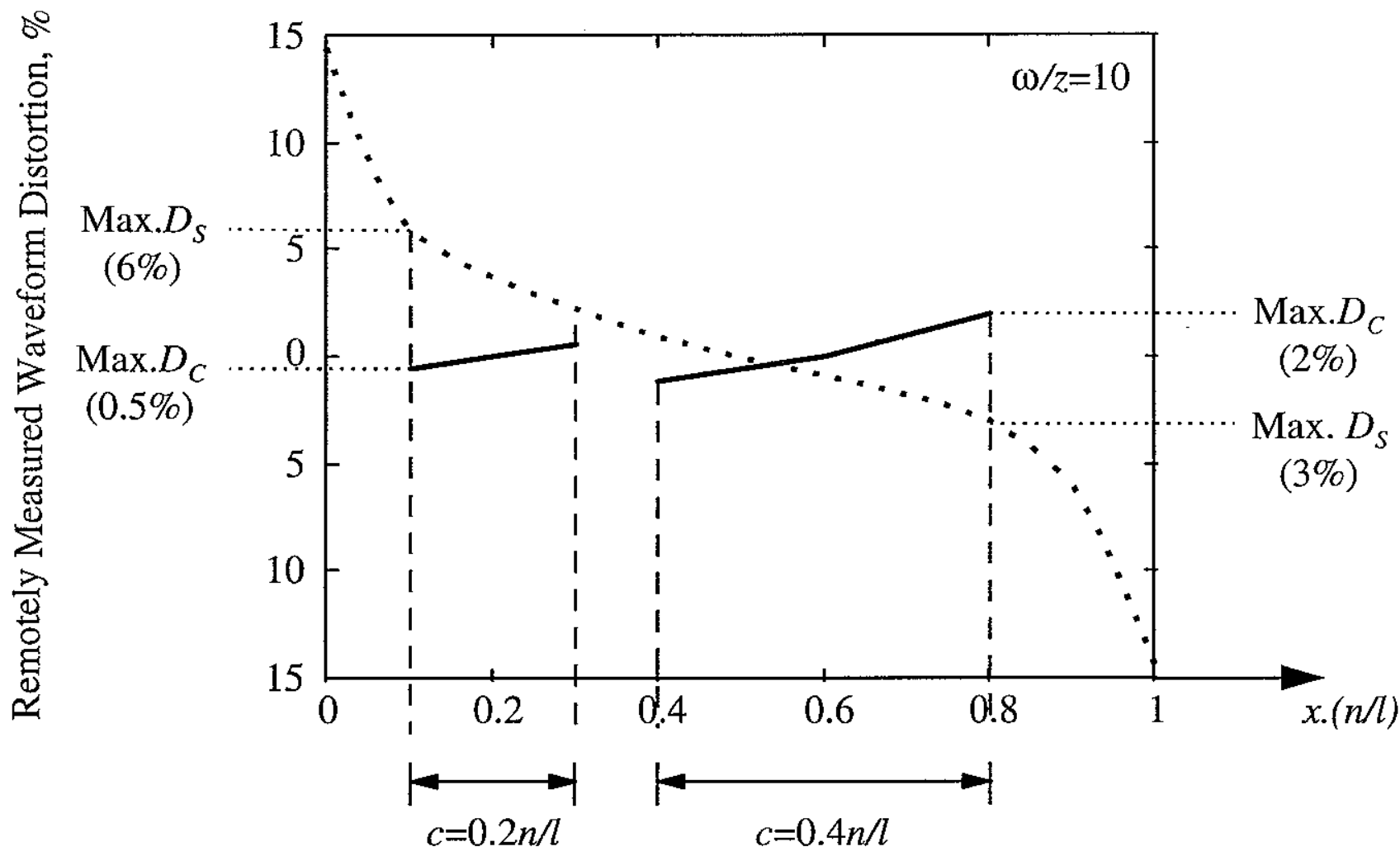

Figure 11

Remote vibration measurements : compensation of waveform distortion due to whole body translations

A.Hocknell, R.Jones \& S.J.Rothberg Loughborough University 


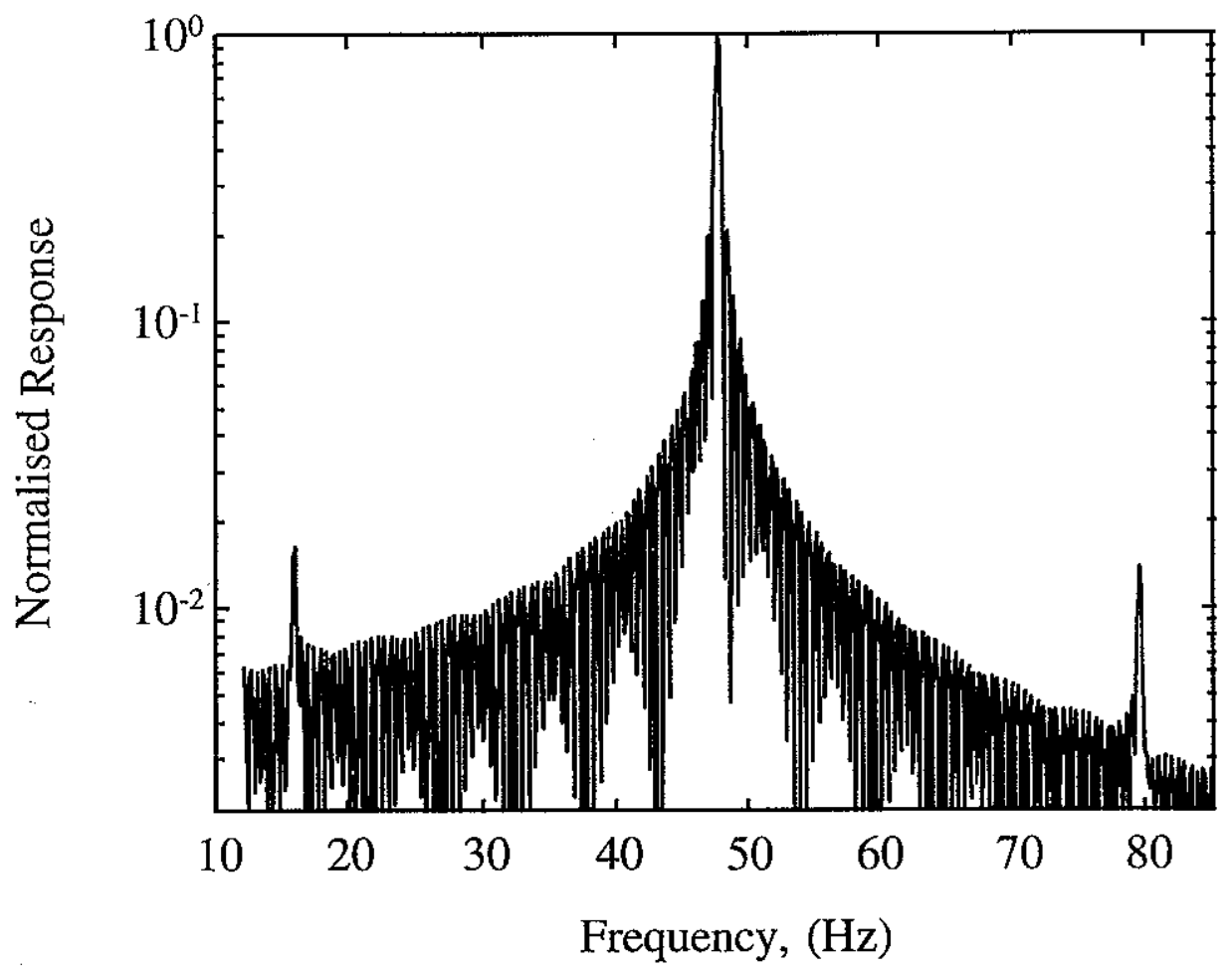

Figure 12

Remote vibration measurements : compensation of waveform distortion due to whole body translations

A.Hocknell, R.Jones \& S.J.Rothberg Loughborough University 


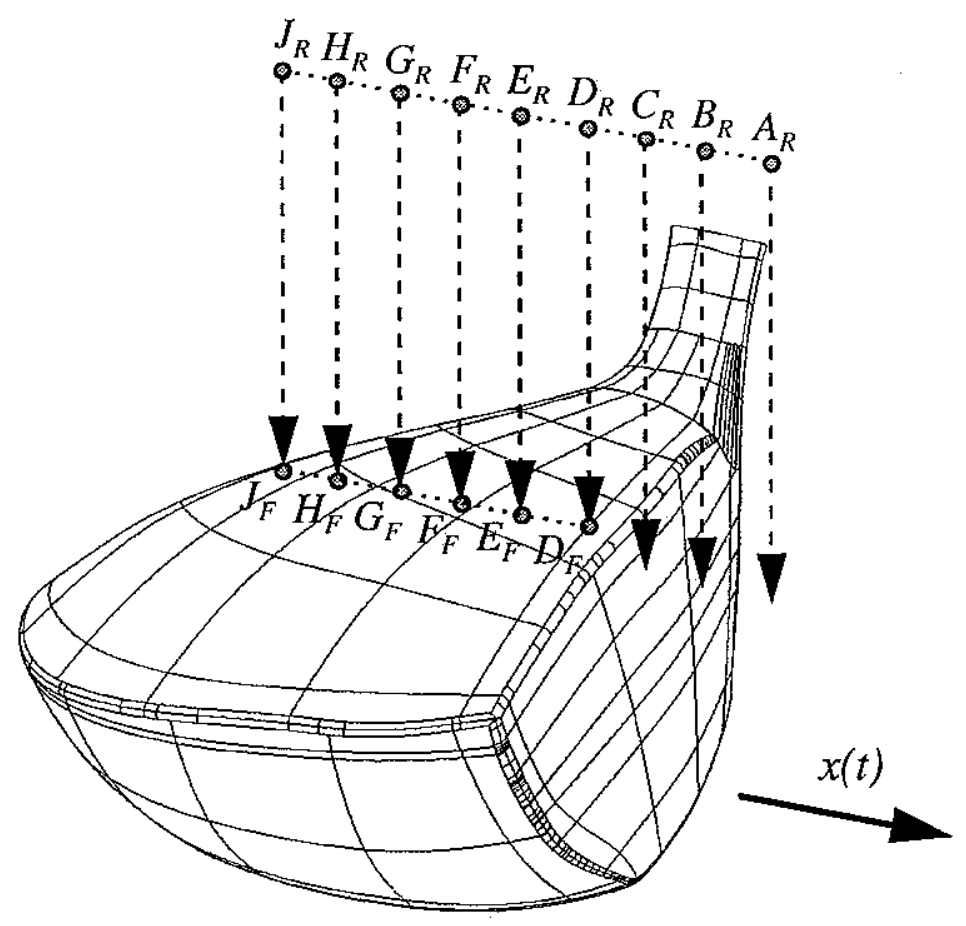

Figure 13

Remote vibration measurements : compensation of waveform distortion due to whole body translations

A.Hocknell, R.Jones \& S.J.Rothberg Loughborough University 


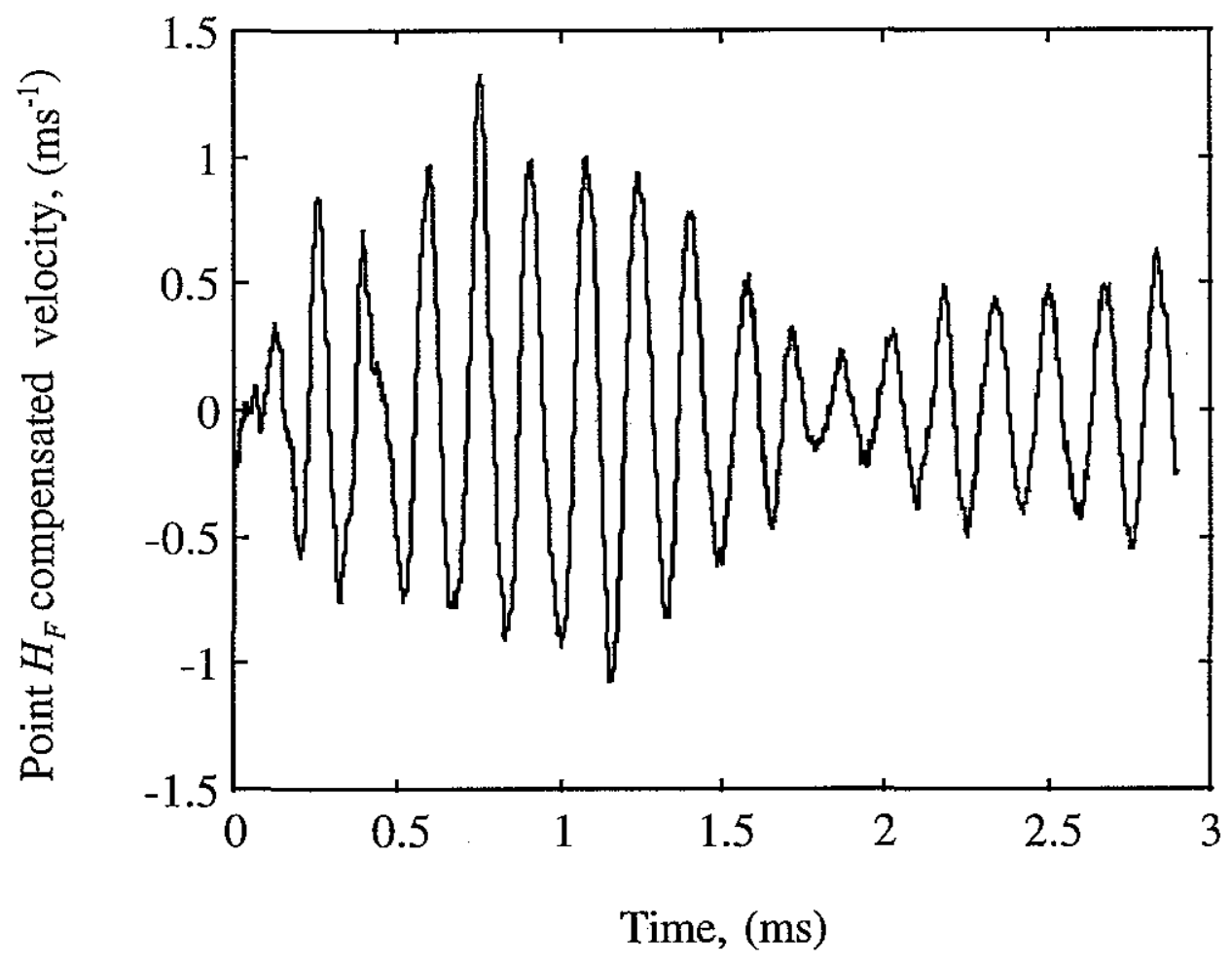

Figure 14

Remote vibration measurements : compensation of waveform distortion due to whole body translations

A.Hocknell, R.Jones \& S.J.Rothberg

Loughborough University 


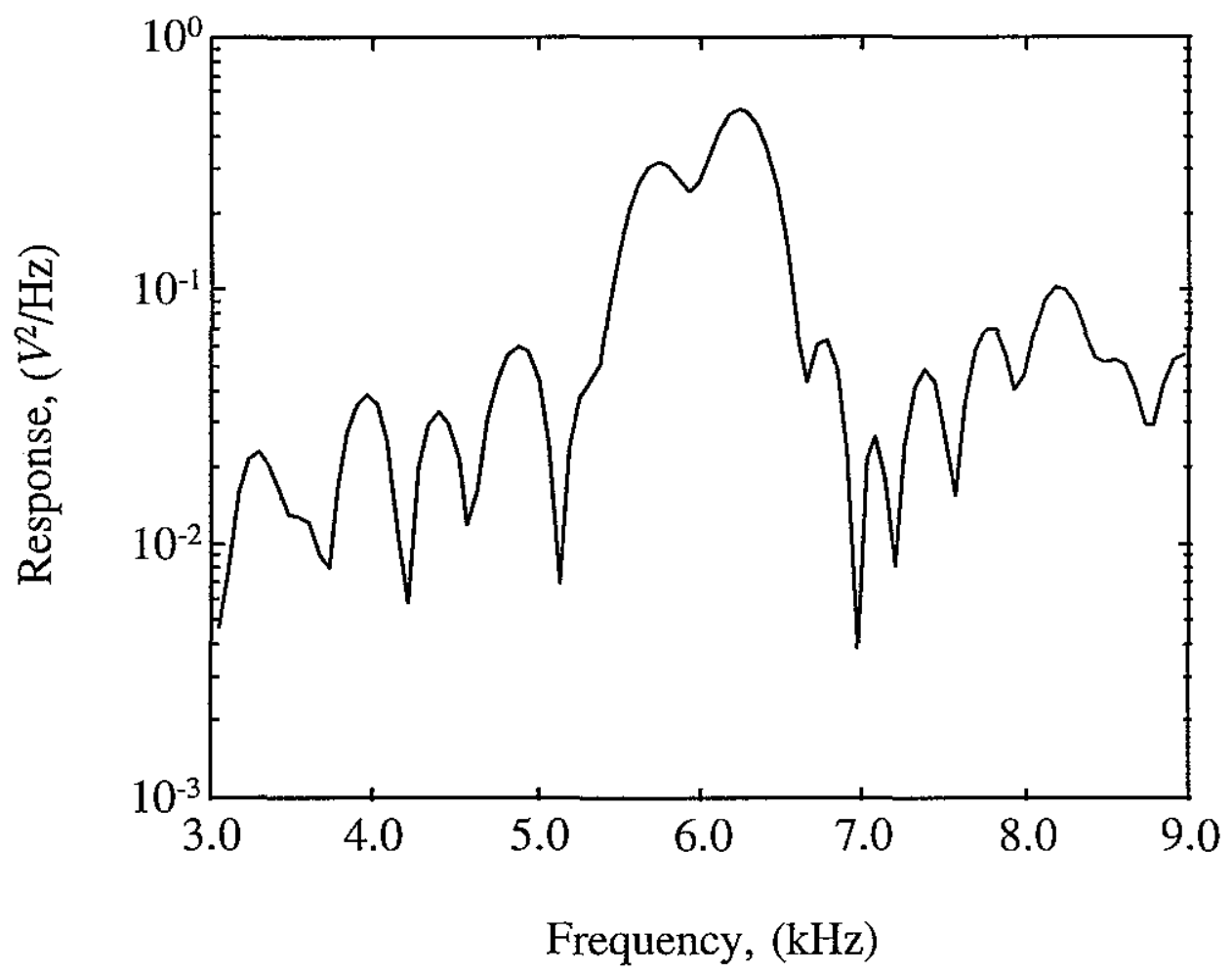

Figure 15

Remote vibration measurements : compensation of waveform distortion due to whole body translations

A.Hocknell, R.Jones \& S.J.Rothberg Loughborough University 


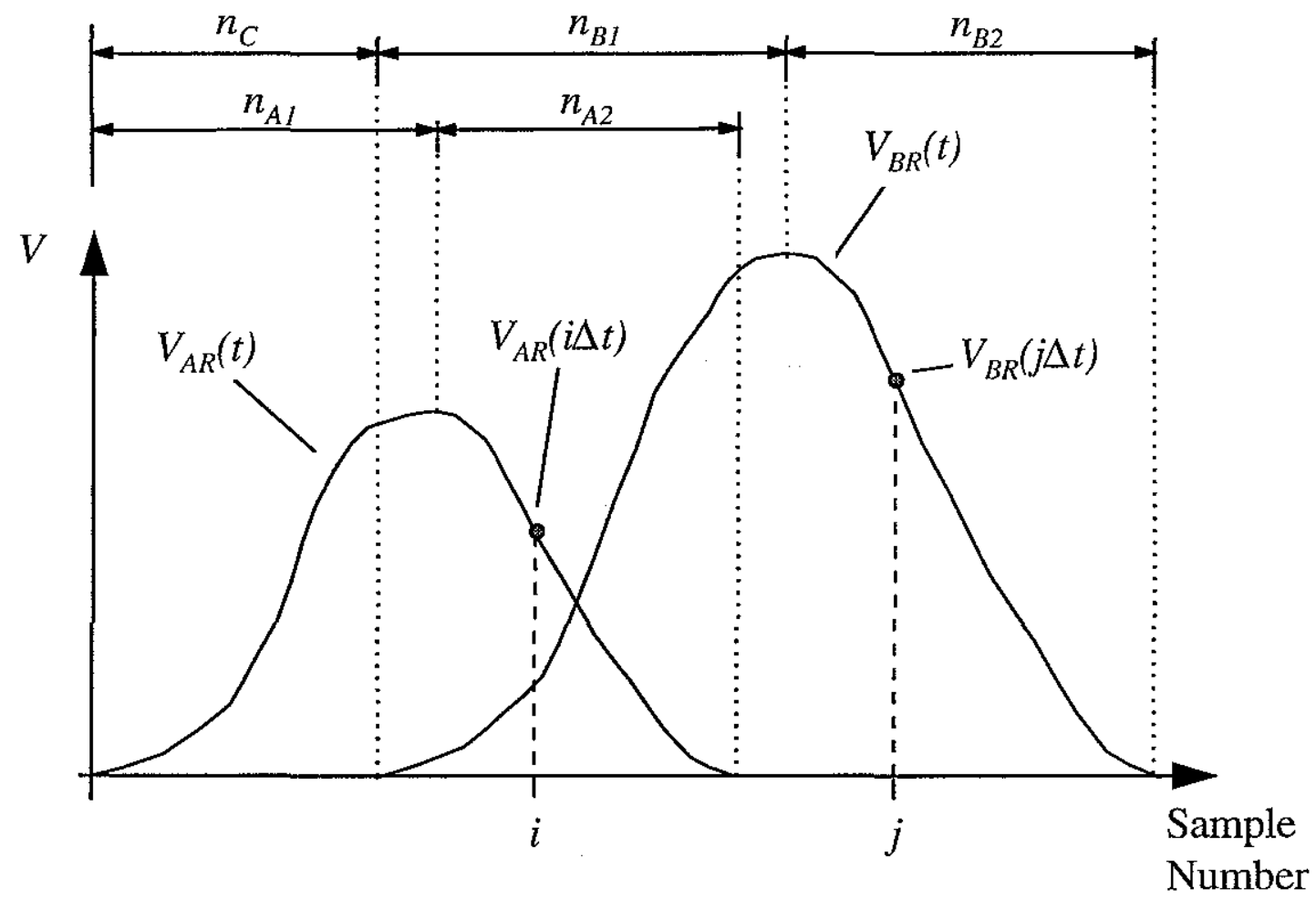

Figure 16a

Remote vibration measurements : compensation of waveform distortion due to whole body translations

A.Hocknell, R.Jones \& S.J.Rothberg Loughborough University 


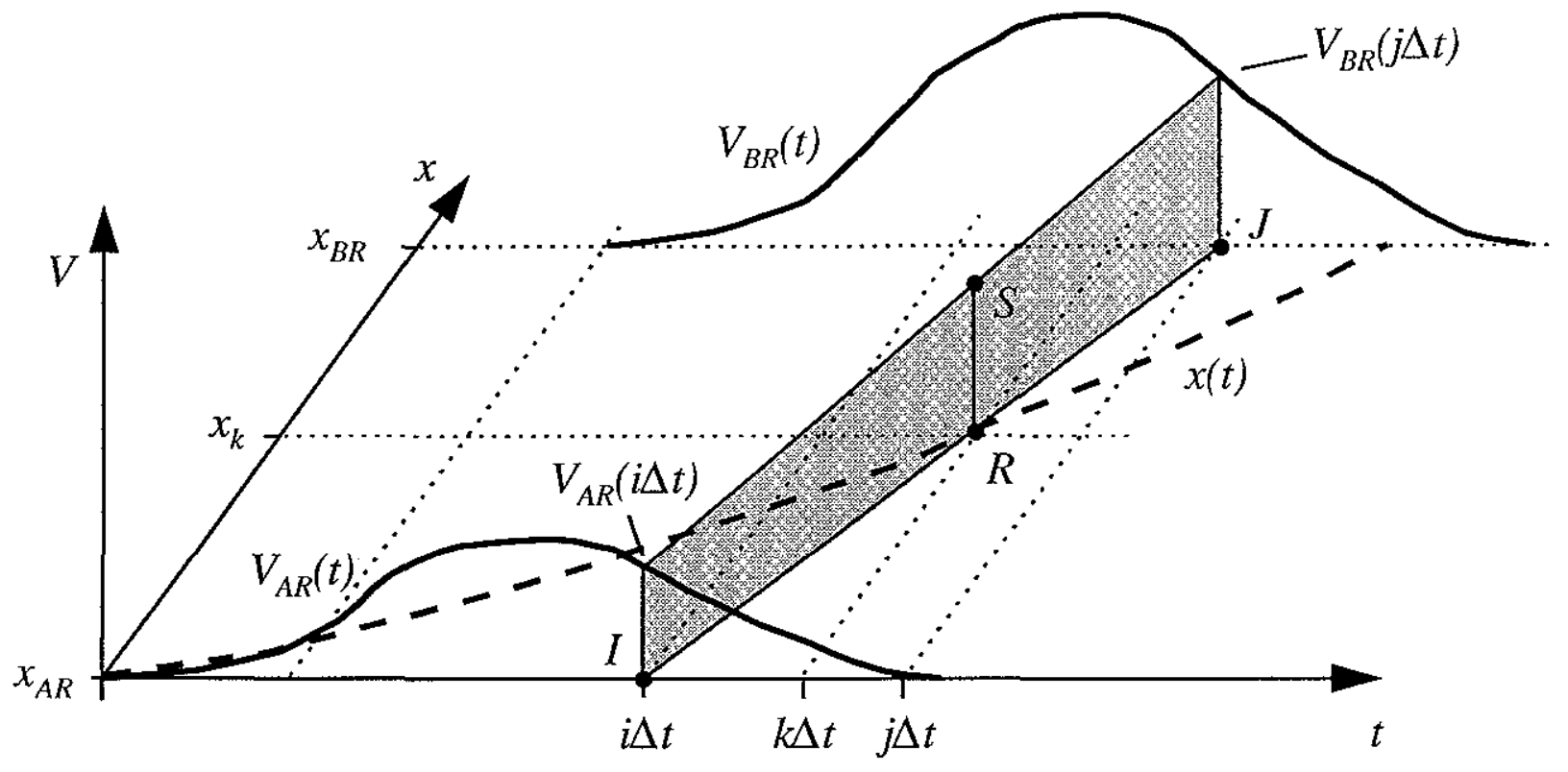

Figure 16b

Remote vibration measurements : compensation of waveform distortion due to whole body translations

A.Hocknell, R.Jones \& S.J.Rothberg Loughborough University 


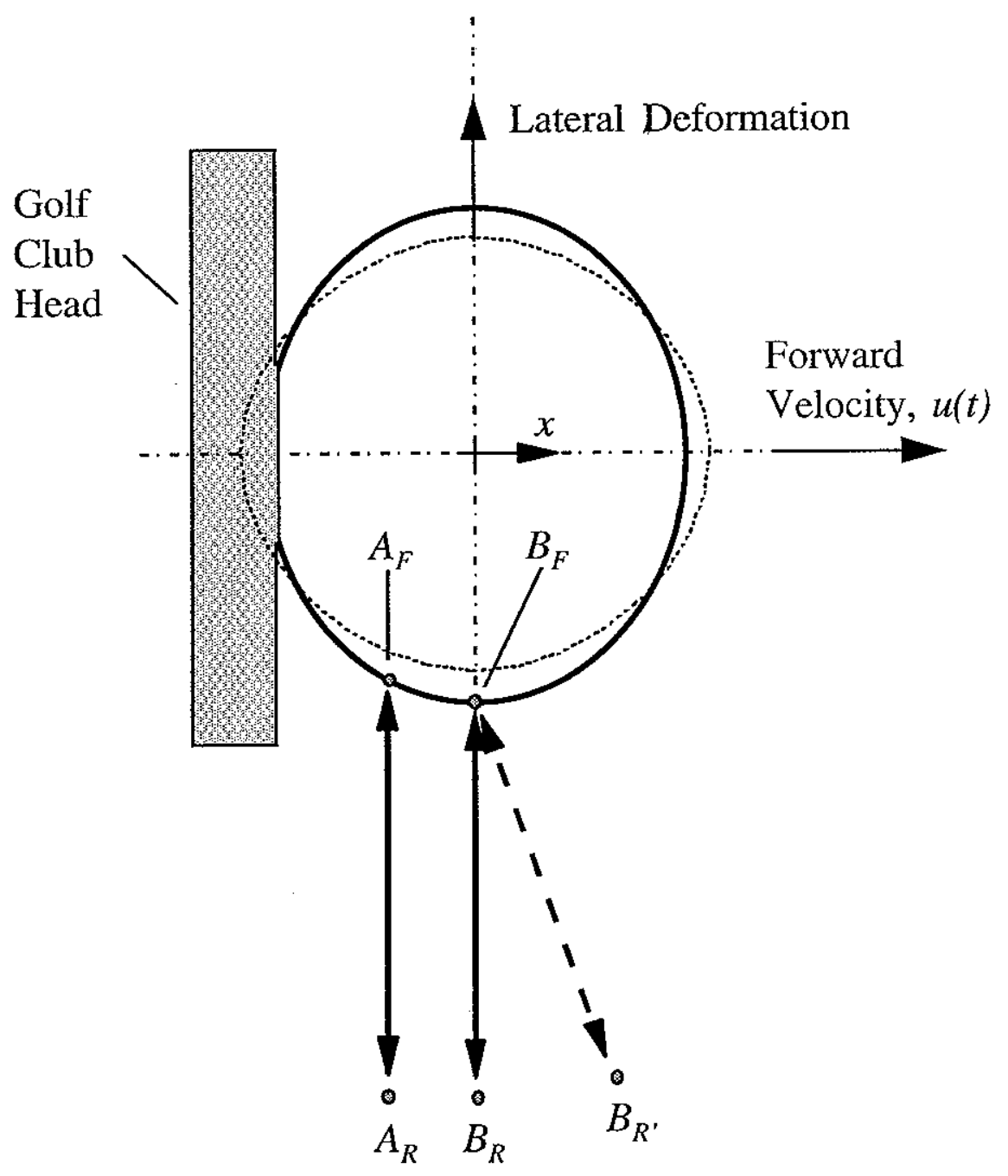

Figure 17

Remote vibration measurements : compensation of waveform distortion due to whole body translations

A.Hocknell, R.Jones \& S.J.Rothberg Loughborough University 


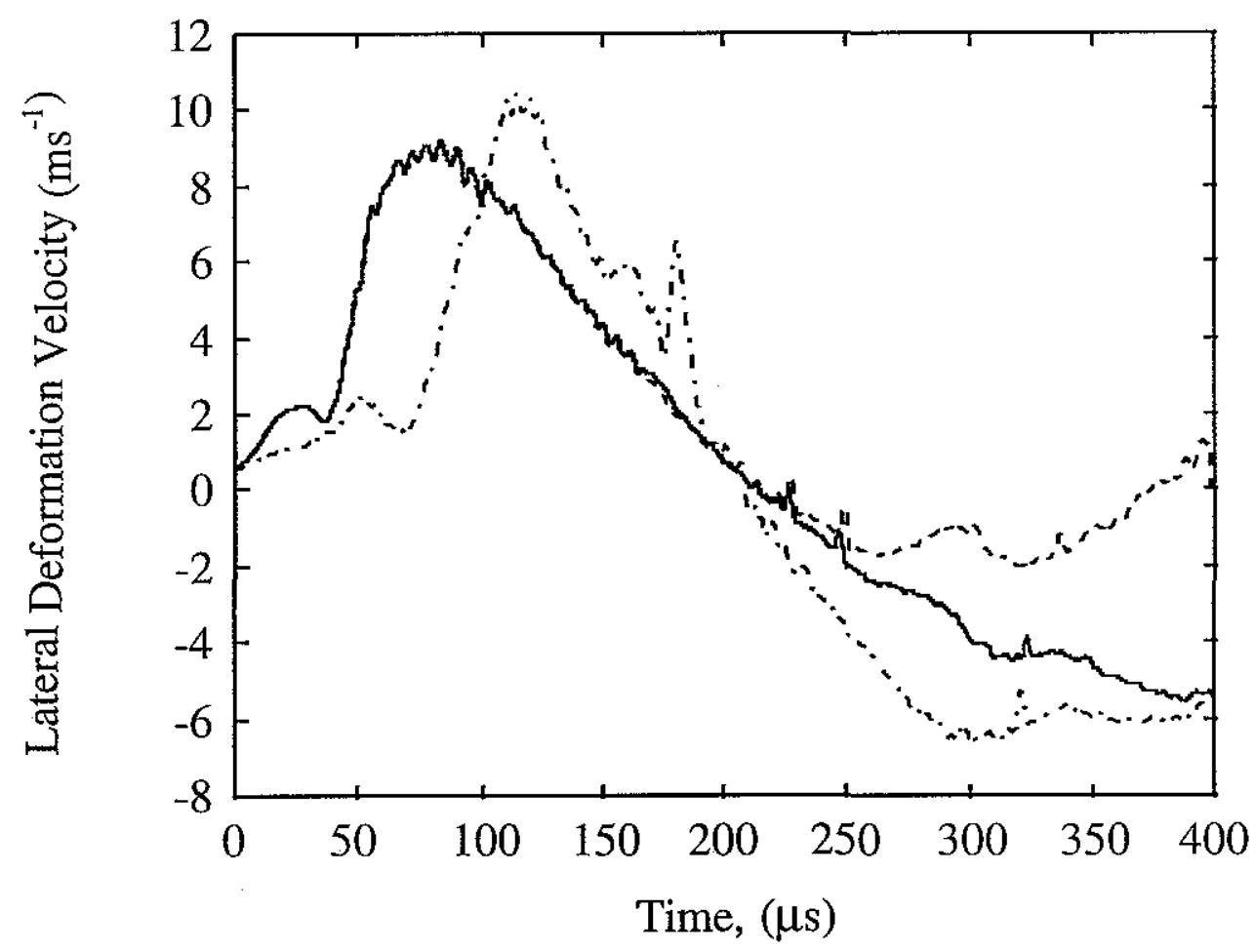

Figure 18

Remote vibration measurements : compensation of waveform distortion due to whole body translations

A.Hocknell, R.Jones \& S.J.Rothberg Loughborough University 